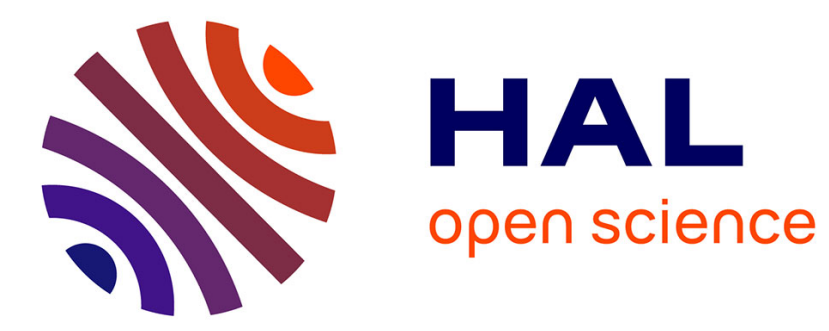

\title{
Reciprocity identities for quasi-static piezoelectric transducer models: Application to cavity identification using iterated excitations and a topological sensitivity approach
}

Cedric Bellis, Sébastien Imperiale

\section{To cite this version:}

Cedric Bellis, Sébastien Imperiale. Reciprocity identities for quasi-static piezoelectric transducer models: Application to cavity identification using iterated excitations and a topological sensitivity approach. Wave Motion, 2013. hal-00875812

\section{HAL Id: hal-00875812 \\ https://hal.inria.fr/hal-00875812}

Submitted on 24 Oct 2013

HAL is a multi-disciplinary open access archive for the deposit and dissemination of scientific research documents, whether they are published or not. The documents may come from teaching and research institutions in France or abroad, or from public or private research centers.
L'archive ouverte pluridisciplinaire HAL, est destinée au dépôt et à la diffusion de documents scientifiques de niveau recherche, publiés ou non, émanant des établissements d'enseignement et de recherche français ou étrangers, des laboratoires publics ou privés. 


\title{
Reciprocity identities for quasi-static piezoelectric transducer models: Application to cavity identification using iterated excitations and a topological sensitivity approach
}

\author{
Cédric Bellis, Sébastien Imperiale \\ Dept of Applied Physics and Applied Mathematics \\ Columbia University, New York, NY 10027, USA
}

\begin{abstract}
The focus of this article is the transient wave-based detection and identification of defects embedded in isotropic elastic solids using piezoelectric transducers. This work addresses this problem within a comprehensive framework encompassing description of elastic wave propagation within the probed media as well as consideration of the coupling phenomena induced by the transducers. A fundamental reciprocity identity associated with a quasi-static piezoelectric model is derived to lay the foundations of ensuing developments and approach of this inverse scattering problem. Modeling of piezoelectric transducers is discussed and application of the proven reciprocity theorem enables the proposition of an iterative construction procedure of electric inputs generating waves expected to focus on the sought defects. The characteristic features of the inverse problem considered, which uses piezoelectric sensor-based measurements, are also discussed. Next, the identification problem is investigated by way of an adjoint field-based topological sensitivity approach that permits the construction of a defect indicator function based on the derived reciprocity identity. For simplicity of exposition, the studied configurations involve defects in the form of traction-free cavities. Finally, a set of $2 \mathrm{D}$ numerical examples based on the spectral finite-elements method is presented to assess the performances of the proposed approach in identifying embedded defects from electric measurements.
\end{abstract}

Keywords. Piezoelectric transducers, Reciprocity identity, Iterated time reversal, Adjoint method, Topological sensitivity.

\section{Introduction}

Transient elastic or ultrasonic waves are preferred phenomena to probe elastic solids in applications such as nondestructive material testing $[34,11]$. Possible embedded and unknown defects, i.e. localized heterogeneities or geometrical features such as cavities or cracks, are illuminated by waves propagating in the solid body considered while 
measurements of their scattered counterparts are collected on a subset of the external surface. Based on such boundary data, a wide range of algorithms aiming at detecting and reconstructing such scattering obstacles have been developed over the last few decades. For instance, optimization-based approaches are generally concerned with the characterization of a finite number of parameters that are quantified by minimization of cost-functionals exploiting the available data, see e.g. [35, 39]. Alternatively, computationally-efficient techniques centered on the construction and the noniterative computation of indicator functions of the sought defects have been more recently developed $[19,31,6]$. These approaches are commonly referred to as qualitative methods [14].

There exists a variety of devices providing full-waveform or partial measurements of boundary displacement fields based on, e.g., mechanical vibrations or electroactive actuators. In this study, it is assumed that the elastic solids of interest are probed using ultrasonic piezoelectric transducers. Such transducers are made of piezoelectric materials which have the property to convert mechanical energy into electric energy, and reciprocally [25]. Here, the piezoelectric phenomena are investigated within the framework of the quasi-static piezoelectric model [27] which features the elastodynamics equation coupled to Maxwell's equations reduced to a scalar electric potential constituting the quantity which can be controlled and recorded during an experiment. In the configuration considered, such a transducer is placed in contact with the investigated elastic medium and it is used both as the source of the illuminating elastic waves as well as, as the receiver of the associated echoes [40, 44]. In emission-mode, customized electric potentials are applied on the active elements of the transducer, which generates a wave propagating in the underlying solid. Alternatively, during the reception regime, or sensor-mode, electric currents associated with the displacement field generated by the mechanical waves impinging the sensor are recorded.

In the present study, the defect identification problem is addressed in a comprehensive framework in dimension two or three, which encompasses description of elastic wave propagation within the probed medium as well as of companion transient piezoelectric phenomena occurring within the transducer. This approach is based on the recent progress concerning the mathematical treatment of piezoelectric transducer models in association with the development of a performant and highly accurate simulation tool [27]. Therefore, the fully-coupled problem arising due to the presence of the piezoelectric transducer is taken into account. Some specific issues arise in this context: Firstly, the presence of the piezoelectric transducer induces an electro-mechanical coupling impacting the elastic field within the probed medium and thus its observation in comparison with a configuration featuring purely elastic boundary excitation and measurements. Moreover, only time-dependent and discrete scalar measurements of the electric field, rather than full-waveform data, are accessible. Lastly, in their most general form, the electric measurements are associated with an integral operator, in time and space, acting on the elastodynamic state associated with the echoes recorded at the sensor's interface with the probed medium. In other words, the mapping between boundary elastic field and measured electric potentials lacks of injectivity and the inverse problem considered is severely ill-posed.

The intended contribution of this work is threefold:

i. Derivation of a time-domain reciprocity identity associated with the quasi-static piezoelectric model considered, a terminology which originates from Betti reciprocity theorem [43]. Improving simplified models such as [20,5] while 
particularizing the studies [30, 32], this identity, which can be seen as stemming from a weak formulation of the problem or the virtual work principle, corresponds to a cross-relation between Neumann and Dirichlet boundary data associated with two solution states satisfying the same piezoelectric field equations over a given geometrical domain. Reciprocitybased methods are classical techniques in the field of non-destructive testing [1], in particular those based on the so-called reciprocity gap concept $[42,13]$. Therefore, the derived identity constitutes a key result for the proposed approach of the inverse problem considered and a thread of the ensuing developments.

ii. Application of the reciprocity theorem to a specific model of piezoelectric transducer, i.e. introducing the set of coupled elastic-electric boundary conditions involved in the emission and reception regimes. This additional result provides the framework for the proposition of an iterative procedure aiming at constructing optimal electric excitations generating elastic waves achieving selective focusing on the unknown defects.

iii. Construction of an indicator function of the sought scattering obstacles which are considered in the form of traction-free cavities for simplicity of exposition. To that purpose, the chosen approach is based on the concept of topological sensitivity which revolves around the quantification of the perturbation of a given cost functional induced by an infinitesimal defect. This method that has been developed and applied in a variety of configurations $[19,12,16,6]$ is extended to the present context of piezoelectric sensor-based measurements by taking advantages of an adjoint-field formulation [10] which requires application of the previously derived reciprocity identities.

This article is organized as follows: The elastic-electric coupled equations describing the behavior of a piezoelectric solid are presented in Section 2, within the framework of a quasi-static approximation of Maxwell's equations. The fundamental result proven in Section 3 is a theorem establishing a reciprocity identity associated with the piezoelectric model considered in a generic geometrical configuration. Section 4 is concerned with the description of a specific model of piezoelectric transducer, application of the derived reciprocity identity and construction of optimally-focusing excitations. Next, the inverse problem considered, i.e. detection and identification of embedded cavities, is stated in Section 5 and the issues arising when dealing with piezoelectric measurements are discussed. Construction of the indicator function along the lines of the topological sensitivity approach is presented Section 6. Finally, numerical results associated with 2D configurations and based on the spectral finite-elements method are shown and discussed in Section 7.

\section{Piezoelectric model}

\subsection{Preliminaries}

Let $\mathbb{R}^{d}$ with dimension $d=2$ or 3 , be endowed with the euclidean scalar product denoted by

$$
\boldsymbol{u} \cdot \boldsymbol{v}=\sum_{i=1}^{d} u_{i} v_{i} \quad \forall(\boldsymbol{u}, \boldsymbol{v}) \in \mathbb{R}^{d} \times \mathbb{R}^{d} .
$$


The space $\mathcal{L}\left(\mathbb{R}^{d}\right)$ of linear mappings from $\mathbb{R}^{d}$ into itself, whose elements are second-order tensors satisfying

$$
(\varepsilon \cdot \boldsymbol{u})_{i}=\sum_{j=1}^{d} \varepsilon_{i j} u_{j} \quad \forall \varepsilon=\left(\varepsilon_{i j}\right) \in \mathcal{L}\left(\mathbb{R}^{d}\right), \forall \boldsymbol{u} \in \mathbb{R}^{d},
$$

is equipped with the scalar product

$$
\boldsymbol{\sigma}: \varepsilon=\sum_{i, j=1}^{d} \sigma_{i j} \varepsilon_{i j}, \quad \forall(\boldsymbol{\sigma}, \boldsymbol{\varepsilon}) \in \mathcal{L}\left(\mathbb{R}^{d}\right) \times \mathcal{L}\left(\mathbb{R}^{d}\right) .
$$

Moreover, let $\mathcal{L}^{2}\left(\mathbb{R}^{d}\right)$ denote the space of linear mappings from $\mathcal{L}\left(\mathbb{R}^{d}\right)$ into itself, in which any element is associated with a fourth-order tensor such as $\mathcal{C}=\left(\mathcal{C}_{i j k l}\right)$ satisfying

$$
(\mathcal{C}: \varepsilon)_{i j}=\sum_{k, l=1}^{d} \mathcal{C}_{i j k l} \varepsilon_{k l}, \quad \forall(\mathcal{C}, \varepsilon) \in \mathcal{L}^{2}\left(\mathbb{R}^{d}\right) \times \mathcal{L}\left(\mathbb{R}^{d}\right) .
$$

Next, let $\mathcal{L}\left(\mathbb{R}^{d}, \mathcal{L}\left(\mathbb{R}^{d}\right)\right)$ denote the space of linear operators from $\mathbb{R}^{d}$ into $\mathcal{L}\left(\mathbb{R}^{d}\right)$, i.e. mapping vectors to second-order tensors. Such operators are associated with third-order tensors such as $\mathbf{d}=\left(\mathrm{d}_{k i j}\right)$ satisfying

$$
(\mathbf{d} \cdot \boldsymbol{u})_{i j}=\sum_{k=1}^{d} \mathrm{~d}_{k i j} u_{k}
$$

The transposed tensor $\mathbf{d}^{\top}$, with respect to the inner products (1) and (2), is an element of the space $\mathcal{L}\left(\mathcal{L}\left(\mathbb{R}^{d}\right), \mathbb{R}^{d}\right)$ of linear mappings from second-order tensors to vectors, and it is defined by

$$
\left(\mathbf{d}^{\top}: \varepsilon\right)_{k}=\sum_{i, j=1}^{d} \mathrm{~d}_{k i j} \varepsilon_{i j} .
$$

The notation div is introduced for the scalar divergence operator which maps vector fields of $\mathbb{R}^{d}$ to real-valued scalars, together with the vectorial divergence div mapping the space $\mathcal{L}\left(\mathbb{R}^{d}\right)$ of tensor fields to $\mathbb{R}^{d}$. Let us recall that

$$
(\operatorname{div} \sigma)_{i}=\operatorname{div}\left(\sigma_{i}\right)
$$

where $\sigma_{i}$ denotes the $i$-th line vector of $\sigma$, i.e. $\sigma_{i}=\sigma^{\top} \cdot \boldsymbol{e}_{i}$ with $\boldsymbol{e}_{i}$ an element of a basis of $\mathbb{R}^{d}$.

To be employed in the ensuing analysis, the Green's formulae on a regular enough domain $\mathcal{O} \subset \mathbb{R}^{d}$ with boundary $\partial \mathcal{O}$, are presented hereafter for the reader's convenience. Given any symmetric second-order tensor $\boldsymbol{\epsilon}(\boldsymbol{x})$, then one has for $(u, v) \in H^{1}(\mathcal{O}) \times H^{1}(\mathcal{O})$

$$
\int_{\mathcal{O}} \operatorname{div}(\boldsymbol{\epsilon} \cdot \nabla u) v \mathrm{~d} \boldsymbol{x}=-\int_{\mathcal{O}} \boldsymbol{\nabla} u \cdot \boldsymbol{\epsilon} \cdot \boldsymbol{\nabla} v \mathrm{~d} \boldsymbol{x}+\int_{\partial \mathcal{O}} \boldsymbol{\epsilon} \cdot \boldsymbol{\nabla} u \cdot \boldsymbol{n} v \mathrm{~d} \sigma
$$

where $\boldsymbol{n}$ is the unit outward normal on $\partial \mathcal{O}$, and for any symmetric fourth-order tensor $\mathcal{C}(\boldsymbol{x})$ and $(\boldsymbol{u}, \boldsymbol{v}) \in H^{1}(\mathcal{O})^{d} \times$ $H^{1}(\mathcal{O})^{d}$ the following identity holds

$$
\int_{\mathcal{O}} \operatorname{div}(\mathcal{C}: \nabla u) \cdot v \mathrm{~d} \boldsymbol{x}=-\int_{\mathcal{O}} \nabla \boldsymbol{u}: \mathcal{C}: \nabla \boldsymbol{v} \mathrm{d} \boldsymbol{x}+\int_{\partial \mathcal{O}} \mathcal{C}: \nabla \boldsymbol{u} \cdot \boldsymbol{n} \cdot \boldsymbol{v} \mathrm{d} \sigma
$$

In the two above identities, the integrals along $\partial \mathcal{O}$ must be understood in the sense of the duality product between $H^{-1 / 2}(\partial \mathcal{O})$ and $H^{1 / 2}(\partial \mathcal{O})$. 


\subsection{Coupled equations of piezoelectricity}

Consider a linear elastic and piezoelectric solid $\Omega_{D} \subset \mathbb{R}^{d}$, with $d=2,3$, not necessarily bounded and isotropic, to be composed of two sub-domains, namely $\Omega_{T}$ which represents the piezoelectric transducer, and $\Omega_{S}^{D}$ the flawed elastic solid to be investigated. Moreover, let $\Omega_{S}$ represent the background medium which is supposed to be known while $D \subset \subset \Omega_{S}$ denotes the geometrical open domain occupied by the compactly contained defect (or the set thereof) to be identified. There exists a variety of possible choices depending on the configuration tested, such as cavity, crack, rigid inclusion or elastic inhomogeneity. As we prefer to keep the presentation simple, the present analysis focuses on traction-free cavities so that the open domain of interest $\Omega_{D}$ is defined by

$$
\overline{\Omega_{D}}=\overline{\Omega_{T}} \cup \overline{\Omega_{S}^{D}}, \quad \text { with } \Omega_{S}^{D}=\Omega_{S} \backslash \bar{D} .
$$

Therefore, since $\operatorname{dist}\left(D, \partial \Omega_{S}\right)>0$ one has $\partial \Omega_{T} \cap \partial \Omega_{S}^{D}=\partial \Omega_{T} \cap \partial \Omega_{S}$. As a reference, the open defect-free domain referred to as $\Omega$ is defined by

$$
\bar{\Omega}=\overline{\Omega_{T}} \cup \overline{\Omega_{S}},
$$

so that $\Omega_{D} \subset \Omega$ for the configurations considered.

When characterizing the linear elastic behavior of solid $\Omega$, let $\rho(\boldsymbol{x})$ denote the mass density, which is a strictly, non-degenerating, positive function, i.e. there exist two positive scalars $\rho_{-}$and $\rho_{+}$such that

$$
0<\rho_{-} \leq \rho(\boldsymbol{x}) \leq \rho_{+}, \quad \text { a.e. } \boldsymbol{x} \in \Omega
$$

Moreover, the so-called elasticity tensor $\mathcal{C}(\boldsymbol{x}) \in \mathcal{L}^{2}\left(\mathbb{R}^{d}\right)$ synthesizes the local elastic material properties at any point $\boldsymbol{x}$ within the domain $\Omega$, and it has the usual major and minor symmetries

$$
\mathcal{C}_{i j k l}=\mathcal{C}_{k l i j}=\mathcal{C}_{j i k l}
$$

The boundedness of the moduli comprising $\mathcal{C}$ together with thermo-mechanical stability conditions also ensure the existence of two scalars $c_{-}$and $c_{+}$such that

$$
0<c_{-}|\varepsilon|^{2} \leq \varepsilon: \mathcal{C}(\boldsymbol{x}): \varepsilon \leq c_{+}|\varepsilon|^{2}, \quad \text { a.e. } \boldsymbol{x} \in \Omega, \quad \forall \varepsilon \in \mathcal{L}\left(\mathbb{R}^{d}\right)
$$

The linearized strain field associated with a displacement field $u \in \mathbb{R}^{d}$ arising in $\Omega_{D}$ or $\Omega$, is defined as the symmetric second-order tensor $\varepsilon[\boldsymbol{u}] \in \mathcal{L}\left(\mathbb{R}^{d}\right)$ such that

$$
(\varepsilon[\boldsymbol{u}])_{i j}=\frac{1}{2}\left(\frac{\partial u_{j}}{\partial x_{i}}+\frac{\partial u_{i}}{\partial x_{j}}\right) .
$$

In the context of a piezoelectric medium, which is characterized by the ability to convert electric energy into mechanical energy, and vice versa, let $\mathbf{d}(x) \in \mathcal{L}\left(\mathbb{R}^{d}, \mathcal{L}\left(\mathbb{R}^{d}\right)\right)$ stand for the so-called piezoelectric tensor synthesizing the corresponding local material properties and which exhibits the following index symmetry

$$
\mathrm{d}_{k i j}=\mathrm{d}_{k j i} .
$$


In the ensuing analysis, the piezoelectric equations are expressed in terms of the scalar electric potential $\varphi$, which is associated with local electric properties that are encapsulated in the second-order permittivity tensor $\boldsymbol{\epsilon}(\boldsymbol{x}) \in \mathcal{L}\left(\mathbb{R}^{d}\right)$ defined in $\mathbb{R}^{d}$, which is assumed to be symmetric, i.e.

$$
\epsilon_{i j}=\epsilon_{j i} .
$$

Moreover, owing to the boundedness of these physical parameters and to the reference vacuum permittivity, their exist two scalars $\epsilon_{-}$and $\epsilon_{+}$such that

$$
0<\epsilon_{-}|\boldsymbol{\psi}|^{2} \leq \boldsymbol{\psi} \cdot \boldsymbol{\epsilon}(\boldsymbol{x}) \cdot \boldsymbol{\psi} \leq \epsilon_{+}|\boldsymbol{\psi}|^{2}, \quad \text { a.e. } \boldsymbol{x} \in \mathbb{R}^{d}, \quad \forall \boldsymbol{\psi} \in \mathbb{R}^{d}
$$

It is also assumed that the active piezoelectric elements consist of an open sub-domain $\Omega_{P} \subset \Omega_{T}$ of the transducer and, as a consequence, the piezoelectric tensor $\mathbf{d}$ vanishes outside $\Omega_{P}$, i.e.

$$
\mathbf{d}(\boldsymbol{x})=\mathbf{0}, \quad \text { a.e. } \boldsymbol{x} \in \mathbb{R}^{d} \backslash \overline{\Omega_{P}} .
$$

When the quasi-static approximation of the Maxwell's equations is valid [25, 27], then the set of coupled piezoelectric field equations in $\Omega$, hereinafter referred to as $\mathfrak{E}(\Omega)$, reduces to

$$
\mathfrak{E}(\Omega): \begin{cases}\rho \frac{\partial^{2} \boldsymbol{u}}{\partial t^{2}}+\alpha \rho \frac{\partial \boldsymbol{u}}{\partial t}-\operatorname{div}(\mathcal{C}: \varepsilon[\boldsymbol{u}])=\operatorname{div}(\mathbf{d} \cdot \nabla \varphi), & \text { in } \Omega, t>0 \\ \operatorname{div}(\boldsymbol{\epsilon} \cdot \boldsymbol{\nabla} \varphi)=\operatorname{div}\left(\mathbf{d}^{\top}: \varepsilon[\boldsymbol{u}]\right), & \text { in } \mathbb{R}^{d}, t>0 .\end{cases}
$$

The positive function $\alpha(\boldsymbol{x})$ is associated with damping properties of the materials constituting the backing (if any) of the piezoelectric transducer considered. Note that the term $\alpha \rho$ featured in the above equation is introduced for consistency with a mass-proportional dissipation, see e.g. [29]. Therefore, it is assumed that there exists a positive scalar $\alpha_{+}$such that

$$
0 \leq \alpha(\boldsymbol{x}) \leq \alpha_{+}, \quad \text { a.e. } \boldsymbol{x} \in \Omega .
$$

Moreover, both the probed elastic solid and the transducer considered are assumed to be initially at rest, so that the initial displacement conditions, formally denoted as $\mathfrak{I}_{\mathfrak{u}}$, reads

$$
\mathfrak{I}_{\mathfrak{u}}: \quad \boldsymbol{u}(\boldsymbol{x}, 0)=\mathbf{0}, \quad \frac{\partial \boldsymbol{u}}{\partial t}(\boldsymbol{x}, 0)=\mathbf{0}, \quad \text { a.e. } \boldsymbol{x} \in \Omega,
$$

while the potential at time $t=0$ satisfies

$$
\mathfrak{I}_{\phi}: \quad \varphi(\boldsymbol{x}, 0)=0 \quad \text { a.e. } \boldsymbol{x} \in \mathbb{R}^{d} .
$$

Note that, if homogeneous electric boundary conditions are provided to complete the problem (4) (see Section 4), then the conditions (5) are sufficient to ensure the homogeneous initial electric condition $\Im_{\phi}$.

Remark 2.1 In the ensuing analysis, the computation of the electric potential $\varphi$ can be reduced to the domain $\Omega_{P}$. This model is mathematically justified in [27] based on the assumption that there exists a high permittivity contrast between 
the piezoelectric elements $\Omega_{P}$ and their surroundings, i.e. providing that the following condition holds

$$
\frac{\sup _{\mathbb{R}^{d} \backslash \overline{\Omega_{P}}} \boldsymbol{\psi} \cdot \boldsymbol{\epsilon} \cdot \boldsymbol{\psi}}{\inf _{\Omega_{P}} \boldsymbol{\psi} \cdot \boldsymbol{\epsilon} \cdot \boldsymbol{\psi}} \ll 1, \quad \forall \boldsymbol{\psi} \in \mathbb{R}^{d} .
$$

Therefore, equation (4b) is restricted to the domain $\Omega_{P}$.

Next, given the geometrical configuration of the problem considered, the exterior surface reads $\partial \Omega=\partial \Omega_{T} \cup \partial \Omega_{S} \backslash$ $\left(\partial \Omega_{T} \cap \partial \Omega_{S}\right)$ and it is assumed to be associated with the coupled traction-free boundary condition

$$
\mathfrak{B}_{\mathfrak{e x t}}: \quad(\mathcal{C}: \varepsilon[\boldsymbol{u}]+\mathbf{d} \cdot \boldsymbol{\nabla} \varphi) \cdot \boldsymbol{n}=\mathbf{0} \quad \text { on the free-surface } \partial \Omega,
$$

with $n$ being the unit outward normal. The elastic boundary conditions are not complete yet in $\Omega_{D}$ as no condition has been specified on the defect boundary $\partial D$. An additional elastic boundary condition denoted $\mathfrak{B}_{\mathfrak{u}}$ is defined as

$$
\mathfrak{B}_{\mathfrak{u}}(\partial D, \boldsymbol{t}): \quad \mathcal{C}: \varepsilon[\boldsymbol{u}] \cdot \boldsymbol{n}=\boldsymbol{t} \quad \text { on } \partial D
$$

with $\boldsymbol{t}=\mathbf{0}$ for the case considered of traction-free cavities.

\section{Reciprocity identity}

The time convolution $[\boldsymbol{u} \star \boldsymbol{v}]$ at time $t \geq 0$ is defined by

$$
[\boldsymbol{u} \star \boldsymbol{v}](\boldsymbol{x}, t)=\int_{0}^{t} \boldsymbol{u}(\boldsymbol{x}, \tau) \otimes \boldsymbol{v}(\boldsymbol{x}, t-\tau) \mathrm{d} \tau .
$$

for generic time-dependent tensor fields $\boldsymbol{u}, \boldsymbol{v}$. Moreover, the combination of time convolution and single (resp. double)

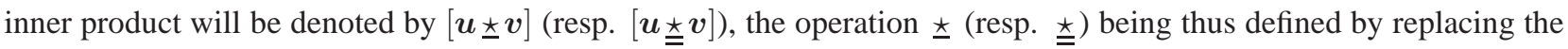
tensor product “ $\otimes$ " sign by the inner product "." (resp. ":”) in the above definition.

We are now in a position to present a key result that is paramount in the ensuing analysis as well as in the construction of a defect indicator function. The following theorem is a reciprocity identity associated with the set (4) of coupled equations that are reduced to a generic open domain $\mathcal{O}$ defined as a subset of the background medium considered and which also includes the transducer, i.e.

$$
\overline{\Omega_{T}} \subset \overline{\mathcal{O}} \subset \bar{\Omega} .
$$

Reference to [1] can be made for an overview on reciprocity theorems in elastodynamics, with a particular application to piezoelectric materials obeying the complete Maxwell's equations. Note that general reciprocity theorems for piezoelectric models that take into account thermo-acoustic and magnetic phenomena are given in [30,32]. Therefore, the theorem provided below can be seen as a particularization of these results to the piezoelectric model considered, which is synthesized by field equations (4) and which also includes material damping. To establish this result, care is taken to describe the featured piezoelectric solutions in the proper functional spaces. 
Theorem 3.1 Let $(\boldsymbol{u}, \varphi)$ and $(\tilde{\boldsymbol{u}}, \tilde{\varphi})$ in $C^{1}\left(\left[0, T_{f}\right] ; L^{2}(\mathcal{O})^{d}\right) \cap C^{0}\left(\left[0, T_{f}\right] ; H^{1}(\mathcal{O})^{d}\right) \times C^{0}\left(\left[0, T_{f}\right] ; H^{1}\left(\Omega_{P}\right)\right)$ satisfying, in a weak sense, the field equations $\mathfrak{E}(\mathcal{O})$ for $t \in\left[0, T_{f}\right]$ as well as the initial condition $\mathfrak{I}_{\mathfrak{u}}$, then these solutions satisfy

$$
\begin{aligned}
& \int_{\partial \mathcal{O}}\{(\mathcal{C}: \varepsilon[\boldsymbol{u}]+\mathbf{d} \cdot \boldsymbol{\nabla} \varphi)\cdot \boldsymbol{n} \underset{\boldsymbol{u}}{\boldsymbol{u}}-(\mathcal{C}: \varepsilon[\tilde{\boldsymbol{u}}]+\mathbf{d} \cdot \boldsymbol{\nabla} \tilde{\varphi}) \cdot \boldsymbol{n} \star \boldsymbol{u}\} \mathrm{d} \sigma \\
&=\int_{\partial \Omega_{P}}\left\{\left(\boldsymbol{\epsilon} \cdot \boldsymbol{\nabla} \varphi-\mathbf{d}^{\top}: \boldsymbol{\varepsilon}[\boldsymbol{u}]\right) \cdot \boldsymbol{n} \star \tilde{\varphi}-\left(\boldsymbol{\epsilon} \cdot \boldsymbol{\nabla} \tilde{\varphi}-\mathbf{d}^{\top}: \boldsymbol{\varepsilon}[\tilde{\boldsymbol{u}}]\right) \cdot \boldsymbol{n} \star \varphi\right\} \mathrm{d} \sigma, \quad t \in\left[0, T_{f}\right] .
\end{aligned}
$$

Proof For simplicity of exposition, a formal derivation of identity (9) is presented based on assumptions of additional regularity, i.e.

$$
(\boldsymbol{u}, \varphi) \text { and }(\tilde{\boldsymbol{u}}, \tilde{\varphi}) \text { in } C^{2}\left(\left[0, T_{f}\right] ; L^{2}(\mathcal{O})^{d}\right) \cap C^{1}\left(\left[0, T_{f}\right] ; H^{1}(\mathcal{O})^{d}\right) \times C^{1}\left(\left[0, T_{f}\right] ; H^{1}\left(\Omega_{P}\right)\right),
$$

while discussion of the interpretation of this relation in the case of minimal regularity is deferred to Remark 3.1.

By taking the convolution and inner product of equation (4a) satisfied by $(\boldsymbol{u}, \varphi)$ with the function $\tilde{\boldsymbol{u}}$, then after integration over the domain $\mathcal{O} \subset \Omega$ one obtains

$$
\int_{\mathcal{O}}\left(\rho \frac{\partial^{2} \boldsymbol{u}}{\partial t^{2}}+\alpha \rho \frac{\partial \boldsymbol{u}}{\partial t}-\operatorname{div}(\mathcal{C}: \varepsilon[\boldsymbol{u}])-\operatorname{div}(\mathbf{d} \cdot \nabla \varphi)\right) \star \tilde{\boldsymbol{u}} \mathrm{d} \boldsymbol{x}=0 .
$$

Using the initial conditions (5) and the properties of the convolution product, the time derivatives are shifted to $\tilde{\boldsymbol{u}}$, i.e.

$$
\int_{\mathcal{O}}\left(\rho \frac{\partial^{2} \boldsymbol{u}}{\partial t^{2}}+\alpha \rho \frac{\partial \boldsymbol{u}}{\partial t}\right) \star \tilde{\boldsymbol{u}} \mathrm{d} \boldsymbol{x}=\int_{\mathcal{O}}\left(\rho \frac{\partial^{2} \tilde{\boldsymbol{u}}}{\partial t^{2}}+\alpha \rho \frac{\partial \tilde{\boldsymbol{u}}}{\partial t}\right) \star \boldsymbol{u} \mathrm{d} \boldsymbol{x} .
$$

The regularity assumptions implies that the $\operatorname{sum} \operatorname{div}(\mathcal{C}: \varepsilon[\boldsymbol{u}])+\operatorname{div}(\mathbf{d} \cdot \nabla \varphi)$ is a square integrable function of space, although this is not true in general for each term of the sum. Nevertheless, for ease of reading, each of these terms are formally handled independently in the next equations.

Using the Green's formula twice, the purely elastic term in (10) is replaced as follows

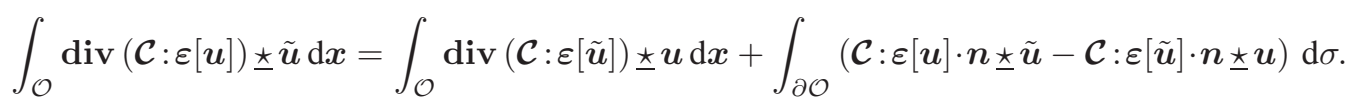

Moreover, the electric term is also recast using Green's formula as

$$
\int_{\mathcal{O}} \operatorname{div}(\mathbf{d} \cdot \nabla \varphi) \star \tilde{\boldsymbol{u}} \mathrm{d} \boldsymbol{x}=-\int_{\mathcal{O}} \boldsymbol{\nabla} \varphi \underline{\underline{ }} \mathbf{d}^{\top}: \varepsilon[\tilde{\boldsymbol{u}}] \mathrm{d} \boldsymbol{x}+\int_{\partial \mathcal{O}}(\mathbf{d} \cdot \nabla \varphi) \cdot \boldsymbol{n}_{\underline{ }} \tilde{\boldsymbol{u}} \mathrm{d} \sigma .
$$

Next, as $\mathbf{d}(\boldsymbol{x})=\mathbf{0}$ when $\boldsymbol{x} \notin \Omega_{P}$ and on noting that $\Omega_{P} \subset \Omega_{T} \subset \mathcal{O}$, then the volume integral at the right-hand side of the previous equation can be restricted to the domain $\Omega_{P}$. Then, application of Green's formula in this subdomain, entails

$$
\int_{\mathcal{O}} \operatorname{div}(\mathbf{d} \cdot \nabla \varphi) \star \tilde{\boldsymbol{u}} \mathrm{d} \boldsymbol{x}=\int_{\Omega_{P}} \operatorname{div}\left(\mathbf{d}^{\top}: \varepsilon[\tilde{\boldsymbol{u}}]\right) \star \varphi \mathrm{d} \boldsymbol{x}-\int_{\partial \Omega_{P}}\left(\mathbf{d}^{\top}: \varepsilon[\tilde{\boldsymbol{u}}]\right) \cdot \boldsymbol{n} \star \varphi \mathrm{d} \sigma+\int_{\partial \mathcal{O}}(\mathbf{d} \cdot \boldsymbol{\nabla} \varphi) \cdot \boldsymbol{n} \star \tilde{\boldsymbol{u}} \mathrm{d} \sigma .
$$

The intermediate equalities (11-13) are then substituted in (10) and the resulting equation is simplified by noticing that $\tilde{\boldsymbol{u}}$ is also solution of (4), which leads to

$$
\begin{aligned}
\int_{\mathcal{O}} \operatorname{div}(\mathbf{d} \cdot \nabla \tilde{\varphi}) \star \boldsymbol{u} \mathrm{d} \boldsymbol{x}-\int_{\Omega_{P}} \operatorname{div}\left(\mathbf{d}^{\top}: \varepsilon[\tilde{\boldsymbol{u}}]\right) \star \varphi \mathrm{d} \boldsymbol{x}= \\
\qquad \int_{\partial \mathcal{O}}\left\{(\mathcal{C}: \varepsilon[\boldsymbol{u}]+\mathbf{d} \cdot \nabla \varphi) \cdot \boldsymbol{n}_{\star} \tilde{\boldsymbol{u}}-\mathcal{C}: \varepsilon[\tilde{\boldsymbol{u}}] \cdot \boldsymbol{n} \star \boldsymbol{u}\right\} \mathrm{d} \sigma-\int_{\partial \Omega_{P}}\left(\mathbf{d}^{\top}: \varepsilon[\tilde{\boldsymbol{u}}]\right) \cdot \boldsymbol{n} \star \varphi \mathrm{d} \sigma .
\end{aligned}
$$


In a similar fashion, by interchanging the roles of $\tilde{\boldsymbol{u}}, \boldsymbol{u}$ and $\tilde{\varphi}, \varphi$ in (13), the term $\operatorname{div}(\mathbf{d} \cdot \nabla \tilde{\varphi}) \star \boldsymbol{u}$ can be replaced accordingly in the previous equation, so that one obtains

$$
\begin{gathered}
\int_{\Omega_{P}}\left\{\operatorname{div}\left(\mathbf{d}^{\top}: \varepsilon[\boldsymbol{u}]\right) \star \tilde{\varphi}-\operatorname{div}\left(\mathbf{d}^{\top}: \varepsilon[\tilde{\boldsymbol{u}}]\right) \star \varphi\right\} \mathrm{d} \boldsymbol{x}= \\
\int_{\partial \mathcal{O}}\left\{(\mathcal{C}: \varepsilon[\boldsymbol{u}]+\mathbf{d} \cdot \nabla \varphi) \cdot \boldsymbol{n}_{\star} \tilde{\boldsymbol{u}}-(\mathcal{C}: \varepsilon[\tilde{\boldsymbol{u}}]+\mathbf{d} \cdot \nabla \tilde{\varphi}) \cdot \boldsymbol{n}_{\star} \boldsymbol{u}\right\} \mathrm{d} \sigma+\int_{\partial \Omega_{P}}\left\{\left(\mathbf{d}^{\top}: \varepsilon[\boldsymbol{u}]\right) \cdot \boldsymbol{n} \star \tilde{\varphi}-\left(\mathbf{d}^{\top}: \varepsilon[\tilde{u}]\right) \cdot \boldsymbol{n} \star \varphi\right\} \mathrm{d} \sigma .
\end{gathered}
$$

The proof is concluded by using equation (4b) for $(\boldsymbol{u}, \varphi)$ and $(\tilde{\boldsymbol{u}}, \tilde{\varphi})$, which finally yields

$$
\begin{aligned}
\int_{\Omega_{P}}\left\{\operatorname{div}\left(\mathbf{d}^{\top}: \varepsilon[\boldsymbol{u}]\right) \star \tilde{\varphi}-\operatorname{div}\left(\mathbf{d}^{\top}: \varepsilon[\tilde{\boldsymbol{u}}]\right) \star \varphi\right\} \mathrm{d} \boldsymbol{x} & =\int_{\Omega_{P}}\{\operatorname{div}(\boldsymbol{\epsilon} \cdot \boldsymbol{\nabla} \varphi) \star \tilde{\varphi} \mathrm{d} \boldsymbol{x}-\operatorname{div}(\boldsymbol{\epsilon} \cdot \boldsymbol{\nabla} \tilde{\varphi}) \star \varphi\} \mathrm{d} \boldsymbol{x} \\
& =\int_{\partial \Omega_{P}}\{(\boldsymbol{\epsilon} \cdot \boldsymbol{\nabla} \varphi \cdot \boldsymbol{n}) \star \tilde{\varphi}-(\boldsymbol{\epsilon} \cdot \boldsymbol{\nabla} \tilde{\varphi} \cdot \boldsymbol{n}) \star \varphi\} \mathrm{d} \sigma .
\end{aligned}
$$

Remark 3.1 It may not be clear mathematically whether the integrated boundary terms at the left-hand side of identity (9), i.e. those involving elastic stresses, exhibit sufficient regularity in time for the convolution products to be defined properly. However, for solutions having minimal regularity, such as weak solutions, then these terms can be recast as volume integrals which actual definitions turn out to be rigorous in a functional sense. More precisely, if $(\boldsymbol{u}, \varphi)$ and $(\tilde{\boldsymbol{u}}, \tilde{\varphi})$ denote solutions belonging to $C^{1}\left(\left[0, T_{f}\right] ; L^{2}(\mathcal{O})^{d}\right) \cap C^{0}\left(\left[0, T_{f}\right] ; H^{1}(\mathcal{O})^{d}\right) \times C^{0}\left(\left[0, T_{f}\right] ; H^{1}\left(\Omega_{P}\right)\right)$ and satisfying, in a weak sense, the field equations $\mathfrak{E}(\mathcal{O})$ for $t \in\left[0, T_{f}\right]$ as well as the initial condition $\mathfrak{I}_{\mathfrak{u}}$ then one has from (4a)

$$
\int_{\partial \mathcal{O}}(\mathcal{C}: \varepsilon[\boldsymbol{u}]+\mathbf{d} \cdot \nabla \varphi) \cdot \boldsymbol{n} \star \tilde{\boldsymbol{u}} \mathrm{d} \sigma \equiv \int_{\mathcal{O}} \rho \frac{\partial \boldsymbol{u}}{\partial t} \star\left(\frac{\partial \tilde{\boldsymbol{u}}}{\partial t}+\alpha \tilde{\boldsymbol{u}}\right) \mathrm{d} \boldsymbol{x}+\int_{\mathcal{O}}(\mathcal{C}: \varepsilon[\boldsymbol{u}]+\mathbf{d} \cdot \nabla \varphi) \underline{\star} \varepsilon[\tilde{\boldsymbol{u}}] \mathrm{d} \boldsymbol{x},
$$

together with the companion equality obtained by interchanging the roles of $\tilde{\boldsymbol{u}}, \boldsymbol{u}$ and $\tilde{\varphi}, \varphi$. In the above identity the right-hand side terms appear to be properly defined, therefore the proven reciprocity relation (9) for such solutions is valid.

\section{Piezoelectric transducer modeling}

\subsection{Geometry and boundary conditions}

In this section, a mathematical model associated with the piezoelectric transducer $\Omega_{T}$ is presented. As a beginning, the transducer geometry to be used in the ensuing analysis is specified. The piezoelectric domain $\Omega_{P} \subset \Omega_{T}$ is assumed to be composed of a number $N_{B}$ of active piezoelectric bars $\Omega_{P}^{i}$ such that

$$
\Omega_{P}=\bigcup_{i=1}^{N_{B}} \Omega_{P}^{i} \quad \text { with } \quad \overline{\Omega_{P}^{i}} \cap \overline{\Omega_{P}^{j}}=\emptyset \text { if } i \neq j .
$$


The external boundary of each bar includes two open surfaces denoted $\Gamma_{i}^{c}$ and $\Gamma_{i}^{a}$, respectively referred to as cathode and anode, and which are characterized by

$$
\partial \Omega_{P}^{i}=\overline{\Gamma_{i}^{c}} \cup \overline{\Gamma_{i}^{a}} \cup \overline{\Gamma_{i}^{r}} \quad \text { with } \quad \Gamma_{i}^{c} \cap \Gamma_{i}^{a}=\Gamma_{i}^{c} \cap \Gamma_{i}^{r}=\Gamma_{i}^{r} \cap \Gamma_{i}^{a}=\emptyset, \quad \Omega_{P}^{i} \text { connected, } \quad \Gamma_{i}^{a} \neq \emptyset,
$$

and $\Gamma_{i}^{r}$ designating the remaining part of $\partial \Omega_{P}^{i}$. Figure 1 describes the standard geometry for the transducer and the underlying solid of interest.

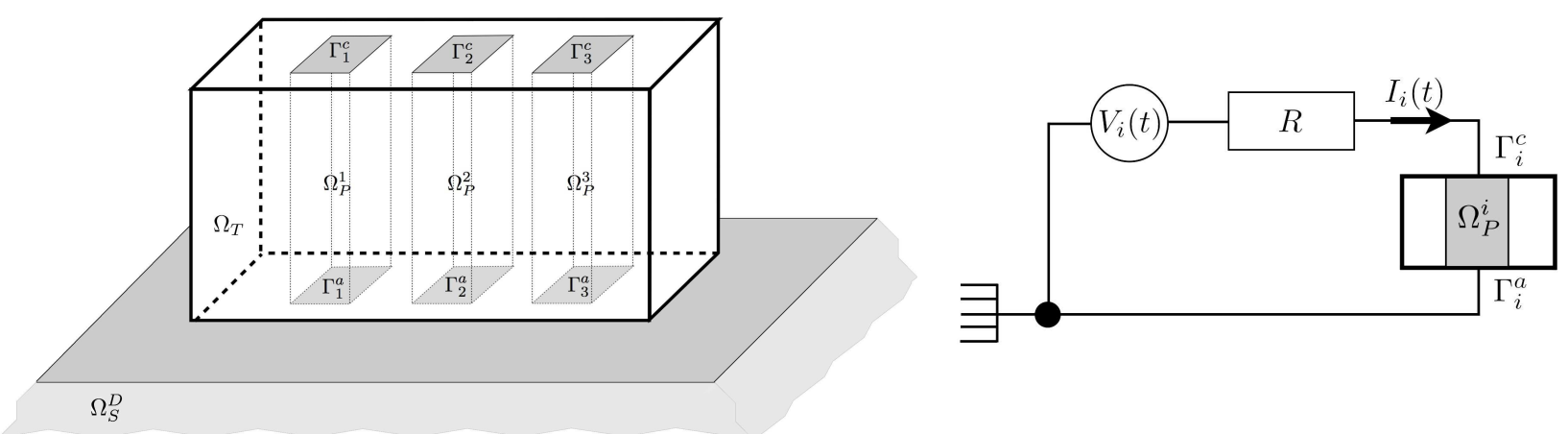

Figure 1: Schematic of transducer geometry with e.g. $N_{B}=3$ (left) and electric circuit between piezoelectric bar $\Omega_{P}^{i}$ and generator (right).

Boundary conditions for the electric potential can now be specified on these different surfaces. On the one hand, for a given bar $\Omega_{P}^{i}$, the boundary condition to be imposed on the corresponding cathode $\Gamma_{i}^{c}$ must allow the different transducer regimes, namely emission and reception modes. On the other hand, the anode $\Gamma_{i}^{a}$ is conventionally grounded at zero potential which is therefore associated with the homogeneous Dirichlet condition

$$
\varphi_{\mid \Gamma_{i}^{a}}=0 .
$$

Moreover, on the remaining bar boundary $\Gamma_{i}^{r}$ and on introducing $\boldsymbol{D}=-\boldsymbol{\epsilon} \cdot \boldsymbol{\nabla} \varphi+\mathbf{d}^{\top}: \varepsilon[\boldsymbol{u}]$ as the so-called electricdisplacement field, then it is proven in [27] that it is a good approximation to consider the following homogeneous mixed boundary condition

$$
\boldsymbol{D} \cdot \boldsymbol{n}=\left(-\boldsymbol{\epsilon} \cdot \boldsymbol{\nabla} \varphi+\mathbf{d}^{\top}: \varepsilon[\boldsymbol{u}]\right) \cdot \boldsymbol{n}=0, \quad \text { on } \bigcup_{i=1}^{N_{B}} \Gamma_{i}^{r},
$$

corresponding to zero normal electric-displacement on $\Gamma_{i}^{r}$ and which completes the set of boundary conditions imposed on $\partial \Omega_{P}^{i}$. Next, in a simplified cathode model [40], the electric charge on the $i$-th cathode $\Gamma_{i}^{c}$ is given by

$$
Q_{i}(t)=-\int_{\Gamma_{i}^{c}} \boldsymbol{D} \cdot \boldsymbol{n} \mathrm{d} \sigma=\int_{\Gamma_{i}^{c}}\left(\boldsymbol{\epsilon} \cdot \boldsymbol{\nabla} \varphi-\mathrm{d}^{\top}: \varepsilon[\boldsymbol{u}]\right) \cdot \boldsymbol{n} \mathrm{d} \sigma,
$$

Each pair of electrodes, i.e. a pair cathode-anode, is connected to a generator (see Figure 1) with common internal resistance $R$ and which imposes an electric potential $V_{i}$ such that the electrode-receiver regime is associated with $V_{i}(t)=$ 0 , while $V_{i}(t) \neq 0$ is e.g. a pulse during the emission mode. Therefore, owing to Ohms' law, one has

$$
V_{i}(t)-\varphi_{\mid \Gamma_{i}^{c}}=R I_{i}(t),
$$


where $I_{i}(t)$ is the electric current flowing into the corresponding piezoelectric bar. Since intensity and electric charge satisfy $I_{i}(t)=d Q_{i}(t) / d t$, then the following mixed boundary condition is finally obtained

$$
\varphi_{\mid \Gamma_{i}^{c}}=V_{i}(t)-R \frac{d}{d t} \int_{\Gamma_{i}^{c}}\left(\boldsymbol{\epsilon} \cdot \nabla \varphi-\mathbf{d}^{\top}: \varepsilon[\boldsymbol{u}]\right) \cdot \boldsymbol{n} \mathrm{d} \sigma .
$$

Then, the coupled electric boundary conditions are synthesized by the following set of equations denoted as $\mathfrak{B}_{\phi}$ :

$$
\mathfrak{B}_{\phi}\left(\left\{V_{i}\right\}\right): \begin{cases}\varphi=V_{i}(t)-R \frac{d}{d t} \int_{\Gamma_{i}^{c}}\left(\boldsymbol{\epsilon} \cdot \boldsymbol{\nabla} \varphi-\mathbf{d}^{\top}: \varepsilon[\boldsymbol{u}]\right) \cdot \boldsymbol{n} \mathrm{d} \sigma, & \text { on } \Gamma_{i}^{c}, i \in\left\{1, \ldots, N_{B}\right\}, t>0, \\ \varphi=0, & \text { on } \Gamma_{i}^{a}, i \in\left\{1, \ldots, N_{B}\right\}, t>0, \\ \left(\boldsymbol{\epsilon} \cdot \boldsymbol{\nabla} \varphi-\mathbf{d}^{\top}: \varepsilon[\boldsymbol{u}]\right) \cdot \boldsymbol{n}=0, & \text { on } \Gamma_{i}^{r}, i \in\left\{1, \ldots, N_{B}\right\}, t>0 .\end{cases}
$$

For fixed time $t$, in order to define the electric potential $\varphi$ in the proper functional space, one introduces

$$
W:=\left\{\psi \in H^{1}\left(\Omega_{P}\right) \mid \psi=0 \text { on } \Gamma_{i}^{a} \text { and } \psi \text { is constant on } \Gamma_{i}^{c} \text { for } i=1, \ldots, N_{B}\right\} .
$$

Therefore, the above space is defined in such way that if $\varphi(\cdot, t) \in W$ then the boundary condition (14a) is well defined while $\varphi(\cdot, t)$ being necessarily constant along $\Gamma_{i}^{c}$.

Remark 4.1 Consider the complete piezoelectric problem constituted by the field equations $\mathfrak{E}(\Omega)$, initial conditions $\mathfrak{I}_{\mathfrak{u}}$ and $\mathfrak{I}_{\phi}$, as well as boundary conditions $\mathfrak{B}_{\mathfrak{e x t}}, \mathfrak{B}_{\mathfrak{u}}$ and $\mathfrak{B}_{\phi}\left(\left\{V_{i}\right\}\right)$ with $\left\{V_{i}(t)\right\}$ in $L^{2}\left(\left[0, T_{f}\right]\right)^{N_{B}}$. Then this mathematical problem lies within a classical functional framework which allows to prove that there exists a unique solution $(\boldsymbol{u}, \varphi)$, see [27], such that

$$
(\boldsymbol{u}, \varphi) \in C^{1}\left(\left[0, T_{f}\right] ; L^{2}(\Omega)^{d}\right) \cap C^{0}\left(\left[0, T_{f}\right] ; H^{1}(\Omega)^{d}\right) \times C^{0}\left(\left[0, T_{f}\right] ; W\right) .
$$

\subsection{Reciprocity identity involving piezoelectric transducer model}

Theorem 3.1 does not specify any particular boundary conditions in terms of displacement or electric potential fields, therefore identity (9) is satisfied in a generic configuration $\mathcal{O} \supset \Omega_{T}$ when the quasi-static approximation of piezoelectricity holds. The following corollary is a direct application of this theorem when the set (14) of boundary conditions is introduced. This situation corresponds to the modeling of a piezoelectric transducer in emission and reception modes.

Corollary 4.1 Consider $(\boldsymbol{u}, \varphi)$ and $(\tilde{\boldsymbol{u}}, \tilde{\varphi})$ in $C^{1}\left(\left[0, T_{f}\right] ; L^{2}(\mathcal{O})^{d}\right) \cap C^{0}\left(\left[0, T_{f}\right] ; H^{1}(\mathcal{O})^{d}\right) \times C^{0}\left(\left[0, T_{f}\right] ; W\right)$ satisfying, in a weak sense, the field equations $\mathfrak{E}(\mathcal{O})$ for $t \in\left[0, T_{f}\right]$, initial conditions $\mathfrak{I}_{\mathfrak{u}}$ and $\mathfrak{I}_{\phi}$, as well as the electric boundary conditions $\mathfrak{B}_{\phi}$ respectively associated with the electric inputs $\left\{V_{i}(t)\right\}$ and $\left\{\tilde{V}_{i}(t)\right\}$ in $L^{2}\left(\left[0, T_{f}\right]\right)^{N_{B}}$.

Then the following identity holds for $t \in\left[0, T_{f}\right]$

$$
\frac{d}{d t} \int_{\partial \mathcal{O}}\left\{(\mathcal{C}: \varepsilon[\boldsymbol{u}]+\mathbf{d} \cdot \nabla \varphi) \cdot \boldsymbol{n}_{\star} \tilde{\boldsymbol{u}}-(\mathcal{C}: \varepsilon[\tilde{\boldsymbol{u}}]+\mathbf{d} \cdot \boldsymbol{\nabla} \tilde{\varphi}) \cdot \boldsymbol{n}_{\star} \boldsymbol{u}\right\} \mathrm{d} \sigma=\frac{1}{R} \sum_{i=1}^{N_{B}}\left(\tilde{\varphi}_{\mid \Gamma_{i}^{c}} \star V_{i}-\varphi_{\mid \Gamma_{i}^{c}} \star \tilde{V}_{i}\right) .
$$


Proof The starting point to prove this corollary is equation (9). The time derivative of the right hand side of (9) is recast as

$$
\begin{aligned}
\mathcal{I}(t):=\frac{d}{d t} \int_{\partial \Omega_{P}}\left\{\left(\boldsymbol{\epsilon} \cdot \boldsymbol{\nabla} \varphi-\mathbf{d}^{\top}: \varepsilon[\boldsymbol{u}]\right) \cdot \boldsymbol{n} \star \tilde{\varphi}-\left(\boldsymbol{\epsilon} \cdot \boldsymbol{\nabla} \tilde{\varphi}-\mathbf{d}^{\top}: \varepsilon[\tilde{\boldsymbol{u}}]\right) \cdot \boldsymbol{n} \star \varphi\right\} \mathrm{d} \sigma \\
=\frac{d}{d t} \sum_{i=1}^{N_{B}} \int_{\Gamma_{i}^{c}}\left\{\left(\boldsymbol{\epsilon} \cdot \boldsymbol{\nabla} \varphi-\mathbf{d}^{\top}: \varepsilon[\boldsymbol{u}]\right) \cdot \boldsymbol{n} \star \tilde{\varphi}-\left(\boldsymbol{\epsilon} \cdot \boldsymbol{\nabla} \tilde{\varphi}-\mathbf{d}^{\top}: \varepsilon[\tilde{\boldsymbol{u}}]\right) \cdot \boldsymbol{n} \star \varphi\right\} \mathrm{d} \sigma,
\end{aligned}
$$

where the homogeneous conditions (14b) and (14c) have been used to get rid of the terms respectively integrated over $\Gamma_{i}^{a}$ and $\Gamma_{i}^{r}$. On noting that, by definition of the space $W, \varphi(\cdot, t)$ and $\tilde{\varphi}(\cdot, t)$ are constant along each $\Gamma_{i}^{c}$ so that they can be removed from the integral over $\Gamma_{i}^{c}$, and using the property of the convolution product with respect to the differentiation when homogeneous initial condition such as $\mathfrak{I}_{\phi}$ holds, one finds

$$
\mathcal{I}(t)=\sum_{i=1}^{N_{B}} \tilde{\varphi} \star \frac{d}{d t} \int_{\Gamma_{i}^{c}}\left(\boldsymbol{\epsilon} \cdot \boldsymbol{\nabla} \varphi-\mathrm{d}^{\mathrm{T}}: \boldsymbol{\varepsilon}[\boldsymbol{u}]\right) \cdot \boldsymbol{n} \mathrm{d} \sigma-\sum_{i=1}^{N_{B}} \varphi \star \frac{d}{d t} \int_{\Gamma_{i}^{c}}\left(\boldsymbol{\epsilon} \cdot \boldsymbol{\nabla} \tilde{\varphi}-\mathrm{d}^{\mathrm{T}}: \varepsilon[\tilde{\boldsymbol{u}}]\right) \cdot \boldsymbol{n} \mathrm{d} \sigma .
$$

This equation is finally simplified using boundary conditions (14a) which finishes the proof.

\subsection{Optimally-focusing excitation}

To probe the flawed solid $\Omega_{S}^{D}$ it is convenient to optimize the illumination generated by the piezoelectric transducer so that focusing is achieved over a region of interest. The energy of the interrogating elastic wave is thus maximized at a prescribed location in order to enhance the signal-to-noise ratio associated with this sampling point. Given the geometry of the transducer $\Omega_{T}$ considered, then the electric inputs $\left\{V_{i}(t)\right\}$ applied on the different piezoelectric bars can feature time-delay parameters $\left\{\tau_{i}\right\}$ chosen so as to obtain focusing at the desired location (see, e.g., [26, 27]). Despite its apparent simplicity, this process relies strongly on the knowledge of the material properties of the transducer and of the underlying medium. Moreover, the focusing points, with associated parameters $\left\{\tau_{i}\right\}$, are chosen a priori, which generally imposes to sample a large region to obtain reliable results. To circumvent these limitations, the proposed approach aims at optimizing the excitation to achieve focusing on the sought $\operatorname{defect}(\mathrm{s}) D$ but without having recourse to any user-chosen parameters. This method finds its roots in the iterative time reversal approach of acoustic fields and the decomposition of the so-called time reversal operator which eigenvectors are associated with waves focusing on the scatterers $[36,38,37,24]$.

Consider the propagation operator for the flawed domain defined as

$$
\begin{aligned}
\mathcal{P}_{D}: & \left\{V_{i}\right\} \in L^{2}\left(\left[0, T_{f}\right]\right)^{N_{B}} \longrightarrow\left\{\varphi_{\mid \Gamma_{i}^{c}}\right\} \in C^{0}\left(\left[0, T_{f}\right]\right)^{N_{B}} \\
& \text { such that }(\boldsymbol{u}, \varphi) \text { satisfies } \mathfrak{E}\left(\Omega_{D}\right), \mathfrak{I}_{\mathfrak{u}}, \mathfrak{I}_{\phi}, \mathfrak{B}_{\mathfrak{e x t}}, \mathfrak{B}_{\mathfrak{u}} \text { and } \mathfrak{B}_{\phi}\left(\left\{V_{i}\right\}\right),
\end{aligned}
$$

with its defect-free counterpart $\mathcal{P}$ associated with the problem $\mathfrak{E}(\Omega)$ in the background domain for which the boundary condition $\mathfrak{B}_{\mathfrak{u}}$ does not hold. As the solution potential $\varphi$ satisfies the following estimates [27]

$$
\sum_{i=1}^{N_{B}}\left\|\varphi_{\mid \Gamma_{i}^{c}}\right\|_{L^{2}\left(\left[0, T_{f}\right]\right)}^{2} \leq T \sum_{i=1}^{N_{B}} \sup _{\left(0, T_{f}\right)}\left(\varphi_{\mid \Gamma_{i}^{c}}\right)^{2} \leq T c_{p} \sum_{i=1}^{N_{B}}\left\|V_{i}\right\|_{L^{2}\left(\left[0, T_{f}\right]\right)}^{2} .
$$


where $c_{p}>0$, then $\mathcal{P}_{D}$ and $\mathcal{P}$ are linear and continuous operators. Next, one introduces the smoothing operator

$$
\mathcal{S}: \quad\left\{\psi_{i}\right\} \in L^{2}\left(\left[0, T_{f}\right]\right)^{N_{B}} \quad \longrightarrow \quad\left\{s \star \psi_{i}\right\} \in H^{1}\left(\left[0, T_{f}\right]\right)^{N_{B}},
$$

where the symbol $s$ is a positive causal time-domain function which Fourier transform $\hat{s}$ satisfies

$$
\left\|\left(1+\omega^{2}\right)^{1 / 2} \hat{s}(\omega)\right\|_{L^{\infty}(\mathbb{R})}=c_{s}<+\infty,
$$

with $c_{s}>0$, which implies, based on Fourier transform properties and Parseval's identity,

$$
\sum_{i=1}^{N_{B}}\left\|s \star \psi_{i}\right\|_{H^{1}\left(\left[0, T_{f}\right]\right)}^{2} \leq c_{s} \sum_{i=1}^{N_{B}}\left\|\psi_{i}\right\|_{L^{2}\left(\left[0, T_{f}\right]\right)}^{2} .
$$

Finally we introduce the time reversal operator

$$
\mathcal{R}: \quad\left\{\psi_{i}(\cdot)\right\} \in C^{0}\left(\left[0, T_{f}\right]\right)^{N_{B}} \quad \longrightarrow \quad\left\{\psi_{i}\left(T_{f}-\cdot\right)\right\} \in C^{0}\left(\left[0, T_{f}\right]\right)^{N_{B}}
$$

Consequently, we consider the composite operator $\mathcal{H}:=\left[\mathcal{R} \mathcal{S}\left(\mathcal{P}_{D}-\mathcal{P}\right)\right]$ which maps electric inputs to the time-reversed counterpart of the regularized potential residual associated with the perturbation induced by the obstacle $\mathrm{D}$ (or the set thereof). Therefore, it is expected that the eigenfunctions of $\mathcal{H}$ that are associated with the eigenvalues of largest amplitude, if they exist, correspond to excitations generating waves that focus on the sought $\operatorname{defect}(\mathrm{s})$. A statement motived by studies $[36,37,24]$. The operator main property is thus characterized by the following theorem which relies strongly on Corollary 4.1 and thus on Theorem 3.1.

Theorem 4.1 The linear operator $\mathcal{H}=\left[\mathcal{R} \mathcal{S}\left(\mathcal{P}_{D}-\mathcal{P}\right)\right]:\left\{V_{i}\right\} \in L^{2}\left(\left[0, T_{f}\right]\right)^{N_{B}} \longrightarrow\left\{\psi_{i}\right\} \in L^{2}\left(\left[0, T_{f}\right]\right)^{N_{B}}$ xadmits a countable orthonormal basis of eigenvectors associated with real eigenvalues accumulating at zero.

Proof One has to prove that $\mathcal{H}$ is (i) self-adjoint and (ii) compact. First one proves (i), i.e. $\mathcal{H}$ is self-adjoint with respect to the $L^{2}$ inner-product in $\left[0, T_{f}\right]$ that is defined by

$$
\left(\left\{\psi_{i}\right\},\left\{\tilde{V}_{i}\right\}\right)_{L^{2}\left(\left[0, T_{f}\right]\right)^{N_{B}}}=\sum_{i=1}^{N_{B}} \int_{0}^{T_{f}} \psi_{i}(t) \tilde{V}_{i}(t) \mathrm{d} t .
$$

On noting $\mathcal{P}_{D}\left(\left\{V_{i}\right\}\right)=\left\{\varphi_{D i}\right\}$ and $\mathcal{P}\left(\left\{V_{i}\right\}\right)=\left\{\varphi_{i}\right\}$ as well as $\mathcal{P}_{D}\left(\left\{\tilde{V}_{i}\right\}\right)=\left\{\tilde{\varphi}_{D i}\right\}$ and $\mathcal{P}\left(\left\{\tilde{V}_{i}\right\}\right)=\left\{\tilde{\varphi}_{i}\right\}$, then one has

$$
\begin{aligned}
\left(\mathcal{H}\left(\left\{V_{i}\right\}\right),\left\{\tilde{V}_{i}\right\}\right)_{L^{2}\left(\left[0, T_{f}\right]\right)^{N_{B}}} & =\sum_{i=1}^{N_{B}} \int_{0}^{T_{f}}\left[s \star\left(\varphi_{D i}-\varphi_{i}\right)\right]\left(T_{f}-t\right) \tilde{V}_{i}(t) \mathrm{d} t \\
& =\left[s \star\left(\sum_{i=1}^{N_{B}}\left(\varphi_{D i}-\varphi_{i}\right) \star \tilde{V}_{i}\right)\right]\left(T_{f}\right)
\end{aligned}
$$

based on the definition of the time reversal operator, the properties of the convolution product and the fact that $\left\{\varphi_{i}\right\}$ are compactly supported and causal functions. Next, owing to definition of operator $\mathcal{P}$ and Corollary 4.1 with the domain $\mathcal{O}$ coinciding with the background geometry, i.e. $\mathcal{O}=\Omega$, one has

$$
\sum_{i=1}^{N_{B}} \varphi_{i} \star \tilde{V}_{i}=\sum_{i=1}^{N_{B}} \tilde{\varphi}_{i} \star V_{i}
$$


using (7). Similarly, definition of $\mathcal{P}_{D}$ together with the piezoelectric reciprocity identity for the configuration $\mathcal{O}=\Omega_{D}$ based on (7) and (8) yield

$$
\sum_{i=1}^{N_{B}} \varphi_{D i} \star \tilde{V}_{i}=\sum_{i=1}^{N_{B}} \tilde{\varphi}_{D i} \star V_{i}-R \frac{d}{d t} \int_{\partial D}\left\{\mathcal{C}: \varepsilon\left[\boldsymbol{u}_{D}\right] \cdot \boldsymbol{n} \star \tilde{\boldsymbol{u}}_{D}-\mathcal{C}: \varepsilon\left[\tilde{\boldsymbol{u}}_{D}\right] \cdot \boldsymbol{n} \star \boldsymbol{u}_{D}\right\} \mathrm{d} \sigma .
$$

where $\boldsymbol{u}_{D}$ and $\tilde{\boldsymbol{u}}_{D}$ are the displacement field solutions of the complete piezoelectric problem in $\Omega_{D}$, respectively associated with the potentials $\left\{\varphi_{D i}\right\}$ and $\left\{\tilde{\varphi}_{D i}\right\}$ on the electrodes. Owing to (8), i.e. the boundary condition $\mathfrak{B}_{\mathfrak{u}}$ imposed on the defect boundary, then the integral along $\partial D$ vanishes in the right-hand side of the previous equation and one finally obtains

$$
\begin{aligned}
\left(\mathcal{H}\left(\left\{V_{i}\right\}\right),\left\{\tilde{V}_{i}\right\}\right)_{L^{2}\left(\left[0, T_{f}\right]\right)^{N_{B}}} & =\left[s \star\left(\sum_{i=1}^{N_{B}}\left(\tilde{\varphi}_{D i}-\tilde{\varphi}_{i}\right) \star V_{i}\right)\right]\left(T_{f}\right) \\
& =\sum_{i=1}^{N_{B}}\left[V_{i} \star\left[s \star\left(\tilde{\varphi}_{D i}-\tilde{\varphi}_{i}\right)\right]\right]\left(T_{f}\right) \\
& =\left(\left\{V_{i}\right\}, \mathcal{H}\left(\left\{\tilde{V}_{i}\right)\right\}\right)_{L^{2}\left(\left[0, T_{f}\right]\right)^{N_{B}}},
\end{aligned}
$$

which proves the self-adjointness of the operator $\mathcal{H}$.

Then one proves (ii), i.e. that $\mathcal{H}$ is a compact operator. Let $\left\{V_{i}\right\}_{n}$ be a bounded sequence in $L^{2}\left(\left[0, T_{f}\right]\right)^{N_{B}}$ with $\left\{\psi_{i}\right\}_{n}=\mathcal{H}\left(\left\{V_{i}\right\}_{n}\right)$, then as a consequence of estimates (16) and (18) one has

$$
\left\|\left\{\psi_{i}\right\}_{n}\right\|_{H^{1}\left(\left[0, T_{f}\right]\right)^{N_{B}}}^{2} \leq T c_{p} c_{s}\left\|\left\{V_{i}\right\}_{n}\right\|_{L^{2}\left(\left[0, T_{f}\right]\right)^{N_{B}}}^{2}, \quad \forall n>1
$$

Therefore, the sequence $\left\{\psi_{i}\right\}_{n}$ is bounded in $H^{1}\left(\left[0, T_{f}\right]\right)^{N_{B}}$ so that by Rellich theorem there exists a subsequence that converges in $L^{2}\left(\left[0, T_{f}\right]\right)^{N_{B}}$ which, by definition, induces the compactness of the operator $\mathcal{H}$.

The proof is concluded by invoking the spectral theorem.

Construction of optimal excitations. Owing to Theorem 4.1, the operator $\left[\mathcal{R} \mathcal{S}\left(\mathcal{P}_{D}-\mathcal{P}\right)\right]$ admits a countable set of orthonormal eigenvectors. Yet, the interpretation of these eigenfunctions are beyond the scope of this study, nonetheless it is expected that the eigenvalues of largest amplitude are associated with eigenfunctions corresponding to waves radiated by the different scatterers present within the probed medium [36, 37, 24]. Therefore, one can iteratively define electric inputs so that the emitted wave will converge to the eigenfunction associated with the eigenvalue of largest amplitude (in the case where its multiplicity is one), and thus targets the strongest scattering obstacle $D$ to be identified.

- Let $\left\{V_{i}\right\}_{0} \in L^{2}\left(\left[0, T_{f}\right]\right)^{N_{B}}$ denote the initial input

- Compute $\left\{W_{i}\right\}_{n}=\left[\mathcal{R} \mathcal{S}\left(\mathcal{P}_{D}-\mathcal{P}\right)\right]\left(\left\{V_{i}\right\}_{n}\right)$

- Define $\left\{V_{i}\right\}_{n}=\left\{W_{i}\right\}_{n} /\left\|\left\{W_{i}\right\}_{n}\right\|_{L^{\infty}\left(\left[0, T_{f}\right]\right)}$

Discussion and assessment of this approach capabilities are deferred to Section 7. Note that this power iteration method can be advantageously superseded by Lanczos iterations [33]. This extension is however left for future work. 
Remark 4.2 The purpose of introducing the smoothing operator $\mathcal{S}$ is twofold: i) From a theoretical standpoint to enforce the compactness of the operator $\mathcal{H}$ and thus the convergence of the above algorithm, and ii) from a practical point of view, to damp the highest frequency phenomena occurring during an experiment or a simulation which could therefore pollute the proposed iterated procedure. In practice, the smoothing function $s(t)$ is chosen as a causal approximation of the Dirac delta function $\delta(t)$.

\section{Measurements and inverse problem}

\subsection{Definition}

The inverse problem considered consists in detecting the unknown defect $D$ and determining qualitatively its topology and geometry. To do so, the domain $\Omega_{S}^{D}$ is illuminated using a given set of source functions $\left\{V_{i}\right\} \in L^{2}\left(\left[0, T_{f}\right]\right)^{N_{B}}$ imposed on the piezoelectric transducer's cathodes. The resultant interrogating electro-mechanical field $\left(\boldsymbol{u}^{\mathrm{obs}}, \varphi^{\mathrm{obs}}\right)$, to be measured, is the solution of the complete piezoelectric problem described by $\mathfrak{E}\left(\Omega_{D}\right), \mathfrak{I}_{\mathfrak{u}}, \mathfrak{I}_{\phi}, \mathfrak{B}_{\mathfrak{e x t}}, \mathfrak{B}_{\mathfrak{u}}(\partial D, \mathbf{0})$ and $\mathfrak{B}_{\phi}\left(\left\{V_{i}\right\}\right)$, i.e. equations (4-8) and (14), and one has

$$
\left(\boldsymbol{u}^{\mathrm{obs}}, \varphi^{\mathrm{obs}}\right) \in C^{1}\left(\left[0, T_{f}\right] ; L^{2}\left(\Omega_{D}\right)^{d}\right) \cap C^{0}\left(\left[0, T_{f}\right] ; H^{1}\left(\Omega_{D}\right)^{d}\right) \times C^{0}\left(\left[0, T_{f}\right] ; W\right) .
$$

To handle the inverse scattering problem considered, it is considered that the only available measurements are the electric potentials on the $N_{B}$ cathodes of the transducer, therefore the defect $D$ has to be characterized from knowledge of

$$
\left\{\varphi^{\mathrm{obs}}{ }_{\mid \Gamma_{i}^{c}}\right\}_{i=1}^{N_{B}} \in C^{0}\left(\left[0, T_{f}\right]\right)^{N_{B}}
$$

\subsection{Measurements and ill-posedness}

Given the nature of measurements (20), a first question concerns the possibility of reconstructing the displacement field $\boldsymbol{u}^{\mathrm{obs}}$ on $\partial \Omega_{T} \cap \partial \Omega_{S}^{D}$. Answering positively this question would ensure the straightforward applicability of existing inversion strategies, in particular of a class of so-called qualitative (or sampling) methods using such remote elastodynamic measurements, see e.g. $[4,31,15,12]$ and the references therein. To address this question, the idea is to establish an explicit relation between $\boldsymbol{u}^{\mathrm{obs}} \mid \partial \Omega_{T} \cap \partial \Omega_{S}^{D}$ and the measured potential $\varphi^{\mathrm{obs}}{ }_{\mid \Gamma_{\ell}^{c}}$ on a given electrode $\Gamma_{\ell}^{c}$ with $\ell \in\left\{1, \ldots, N_{B}\right\}$.

Let $\left(\boldsymbol{G}_{\ell}(\boldsymbol{x}, t), \Phi_{\ell}(\boldsymbol{x}, t)\right)$ denote the fundamental piezoelectric solution associated with the electrode $\Gamma_{\ell}^{c}$, defined as the solution of the problem $\mathfrak{E}\left(\Omega_{T}\right)$, i.e.

$$
\begin{cases}\rho \frac{\partial^{2} \boldsymbol{G}_{\ell}}{\partial t^{2}}+\alpha \rho \frac{\partial \boldsymbol{G}_{\ell}}{\partial t}-\operatorname{div}\left(\mathcal{C}: \varepsilon\left[\boldsymbol{G}_{\ell}\right]\right)=\operatorname{div}\left(\mathbf{d} \cdot \nabla \Phi_{\ell}\right), & \text { in } \Omega_{T}, t>0, \\ \operatorname{div}\left(\boldsymbol{\epsilon} \cdot \nabla \Phi_{\ell}\right)=\operatorname{div}\left(\mathbf{d}^{\top}: \varepsilon\left[\boldsymbol{G}_{\ell}\right]\right), & \text { in } \Omega_{P}, t>0\end{cases}
$$


together with electric boundary conditions $\mathfrak{B}_{\phi}\left(\left\{V_{i}\right\}\right)$ with source $V_{i}(t)=\delta_{i \ell} \delta(t)$

$$
\begin{cases}\Phi_{\ell}=\delta_{i \ell} \delta(t)-R \frac{d}{d t} \int_{\Gamma_{i}^{c}}\left(\boldsymbol{\epsilon} \cdot \nabla \Phi_{\ell}-\mathbf{d}^{\mathrm{T}}: \varepsilon\left[\boldsymbol{G}_{\ell}\right]\right) \cdot \boldsymbol{n} \mathrm{d} \sigma, & \text { on } \Gamma_{i}^{c}, i \in\left\{1, \ldots, N_{B}\right\}, t>0, \\ \Phi_{\ell}=0, & \text { on } \Gamma_{i}^{a}, i \in\left\{1, \ldots, N_{B}\right\}, t>0, \\ \left(\boldsymbol{\epsilon} \cdot \boldsymbol{\nabla} \Phi_{\ell}-\mathbf{d}^{\mathrm{\top}}: \varepsilon\left[\boldsymbol{G}_{\ell}\right]\right) \cdot \boldsymbol{n}=0, & \text { on } \Gamma_{i}^{r}, i \in\left\{1, \ldots, N_{B}\right\}, t>0,\end{cases}
$$

which corresponds to the situation where the $\ell$-th electrode has been excited by an electric Dirac delta function. To define completely $\left(\boldsymbol{G}_{\ell}(\boldsymbol{x}, t), \Phi_{\ell}(\boldsymbol{x}, t)\right)$ the following mixed elastic boundary conditions are added:

$$
\left(\mathcal{C}: \varepsilon\left[\boldsymbol{G}_{\ell}\right]+\mathbf{d} \cdot \boldsymbol{\nabla} \Phi_{\ell}\right) \cdot \boldsymbol{n}=\mathbf{0} \quad \text { on } \partial \Omega_{T} \backslash\left(\partial \Omega_{T} \cap \partial \Omega_{S}\right) \quad \text { and } \quad \boldsymbol{G}_{\ell}=\mathbf{0} \quad \text { on } \partial \Omega_{T} \cap \partial \Omega_{S},
$$

which are conditions satisfied at the transducer boundary, together with homogeneous initial conditions $\mathfrak{I}_{\mathfrak{u}}$ and $\mathfrak{I}_{\phi}$. Note again that in the configuration of interest which involves an internal defect, one has $\partial \Omega_{T} \cap \partial \Omega_{S}^{D}=\partial \Omega_{T} \cap \partial \Omega_{S}$.

Remark 5.1 The fundamental piezoelectric solution considered can be interpreted as the limit solution obtained using parametrized and uniformly bounded source terms $V_{i}(t, \eta) \in L^{1}\left(\left[0, T_{f}\right]\right)$ with $V_{i}(t, \eta) \rightarrow \delta_{i \ell} \delta(t)$ when $\eta \rightarrow 0$.

Existence and uniqueness results can be shown, and one has that the solution $\left(\boldsymbol{G}_{\ell}(\boldsymbol{x}, t), \Phi_{\ell}(\boldsymbol{x}, t)\right)$ can be used to define double-layer potential representations of the piezoelectric solutions. Now, applying, formally, Corollary 4.1 to $\left(\boldsymbol{G}_{\ell}(\boldsymbol{x}, t), \Phi_{\ell}(\boldsymbol{x}, t)\right)$ and $\left(\boldsymbol{u}^{\text {obs }}, \varphi^{\text {obs }}\right)$ in $\mathcal{O}=\Omega_{T}$ leads to

$$
\varphi^{\mathrm{obs}}{ }_{\mid \Gamma_{\ell}^{c}}=\sum_{i=1}^{N_{B}} \Phi_{\ell \mid \Gamma_{i}^{c}} \star V_{i}+R \frac{d}{d t} \int_{\partial \Omega_{T} \cap \partial \Omega_{S}}\left(\mathcal{C}: \varepsilon\left[\boldsymbol{G}_{\ell}\right]+\mathbf{d} \cdot \nabla \Phi_{\ell}\right) \cdot \boldsymbol{n} \boldsymbol{u}^{\mathrm{obs}} \mathrm{d} \sigma,
$$

which characterizes the measured electric potential at the electrode $\ell \in\left\{1, \ldots, N_{B}\right\}$ as a double-layer potential having the displacement field $\boldsymbol{u}^{\text {obs }}$ on $\partial \Omega_{T} \cap \partial \Omega_{S}$ as its density.

The measurements (20) considered, consist of a number of $N_{B}$ time-dependent scalar functions $\varphi^{\text {obs }}$ on $\Gamma_{\ell}^{c}$ corresponding to a time-dependent spatial average of the three-dimensional vector field $\boldsymbol{u}^{\text {obs }}$ at the interface $\partial \Omega_{T} \cap \partial \Omega_{S}^{D}$ between the probed domain and the sensor. Therefore, the displacement $\boldsymbol{u}^{\mathrm{obs}}{ }_{\mid \partial \Omega_{T} \cap \partial \Omega_{S}^{D}}$ on the observation surface cannot be reconstructed uniquely from the knowledge of the electric potential $\varphi^{\text {obs }}$ on all the electrodes. For example, the measurements are invariant with respect to the geometrical symmetries of the sensor considered (classically a cylinder). More precisely, on using previous representation (21), ascertaining whether or not the mapping between elastic field and measurements is injective reduces to characterizing the null-space of the integral operator $K$ defined by

$$
\begin{aligned}
K: & \boldsymbol{u} \in C^{0}\left(\left[0, T_{f}\right], H^{1 / 2}\left(\partial \Omega_{T} \cap \partial \Omega_{S}\right)^{d}\right) \quad \longrightarrow \quad K \boldsymbol{u} \in C^{0}\left(\left[0, T_{f}\right]\right)^{N_{B}} \\
& \text { such that }(K \boldsymbol{u})_{\ell}=\int_{\partial \Omega_{T} \cap \partial \Omega_{S}}\left(\mathcal{C}: \boldsymbol{\varepsilon}\left[\boldsymbol{G}_{\ell}\right]+\mathbf{d} \cdot \boldsymbol{\nabla} \Phi_{\ell}\right) \cdot \boldsymbol{n} \star \boldsymbol{u} \mathrm{d} \sigma, \quad \ell \in\left\{1, \ldots, N_{B}\right\},
\end{aligned}
$$

where $\boldsymbol{u}$ is solution of the fully coupled piezoelectric problem.

One can conclude that the measurements (20) at hand in the problem considered are markedly poor in terms of available informations for the following reasons: i) The use of the piezoelectric transducer induces an electro-mechanical 
coupling which impacts the observation of the field scattered by the sought obstacle $D$ compared to a configuration employing purely elastic boundary conditions and excitation. ii) The measurements involve remote boundary observations on $\partial \Omega_{S}^{D}$ and spatial averaging of the probing elastodynamic state $\boldsymbol{u}^{\text {obs }}$ arising in $\Omega_{S}^{D} \subset \Omega_{D}$. iii) Only time-dependent scalar, rather than vectorial, quantities are available. To circumvent these limitations, the approach proposed in [8] consists in considering the identification problem within the limit geometric configuration of a slender piezoelectric bar, for which an explicit relation between the measured electric potential and the elastic displacement field at $\partial \Omega_{T} \cap \partial \Omega_{S}$ can be established by asymptotic analysis.

\section{Topological sensitivity approach}

This section concerns the construction of a defect indicator function based on the concept of topological sensitivity and on an adjoint-field based approach that uses the derived reciprocity identity (15) of Corollary 4.1.

\subsection{Presentation}

Identification of the defect $D$ is based on the availability of electric potential measurements of the form (20) during the time interval $\left[0, T_{f}\right]$ and corresponding to a coupled probing elastodynamic-electric state $\left(\boldsymbol{u}^{\mathrm{obs}}, \varphi^{\mathrm{obs}}\right)$ arising in the flawed solid and the transducer due to electric excitation prescribed on the electrodes. The discrepancy between a trial configuration $\Omega_{D}^{*}$ and the unknown domain $\Omega_{D}$ is evaluated by means of a cost functional $\mathbb{J}$ in terms of a given misfit density function. Such a misfit function is chosen so as to evaluate the gap between observations $\varphi^{\mathrm{obs}}{ }_{\mid \Gamma_{i}^{c}}$ and measurements $\varphi^{*}{ }_{\mid \Gamma_{i}^{c}}$ of the potential solution associated with the trial flawed solid $\Omega_{D}^{*}$. Numerical experiments presented in this work are based on a commonly-used least squares misfit function, i.e.

$$
\mathbb{J}\left(\varphi^{*} ; T_{f}\right)=\frac{1}{2} \int_{0}^{T_{f}} \sum_{i=1}^{N_{B}}\left|\varphi_{\mid \Gamma_{i}^{c}}^{*}-\varphi_{\mid \Gamma_{i}^{c}}^{\mathrm{obs}}\right|^{2} \mathrm{~d} t,
$$

where the experiment duration $T_{f}$ appears as an adjustable parameter.

The topological sensitivity of the cost functional (23) is here defined as its sensitivity with respect to the creation of an infinitesimal traction-free obstacle of characteristic size $a$ at a given location $z \in \Omega_{S}$ and formally defined by $D_{a, \boldsymbol{z}}=\boldsymbol{z}+a \mathcal{D}$ in terms of a characteristic radius $a>0$ and a normalized open surface $\mathcal{D}$ containing the origin and specifying a chosen shape (e.g. $\mathcal{D}$ is a unit sphere for a nucleating spherical cavity in $\mathbb{R}^{3}$ ). The corresponding trial open flawed domain is defined such that

$$
\overline{\Omega_{D}^{*}}:=\overline{\Omega_{a, \boldsymbol{z}}}=\overline{\Omega_{T}} \cup \overline{\left(\Omega_{S} \backslash \overline{D_{a, z}}\right)} .
$$

In $\Omega_{a, \boldsymbol{z}}$, the prescribed electric excitation gives rise to a coupled state $\left(\boldsymbol{u}_{a, \boldsymbol{z}}, \varphi_{a, \boldsymbol{z}}\right)$ that can be conveniently decomposed by linearity into $\boldsymbol{u}_{a, \boldsymbol{z}}=\boldsymbol{u}_{\mid \Omega_{a, z}}+\boldsymbol{v}_{a, z}$ and $\varphi_{a, \boldsymbol{z}}=\varphi_{\mid \Omega_{a, z}}+\psi_{a, \boldsymbol{z}}$, where the free-field solution $(\boldsymbol{u}, \varphi)$ is the response of the reference defect-free domain $\Omega$, i.e.

$$
(\boldsymbol{u}, \varphi) \quad \text { satisfies } \quad \mathfrak{E}(\Omega), \mathfrak{I}_{\mathfrak{u}}, \mathfrak{I}_{\phi}, \mathfrak{B}_{\mathfrak{e x t}} \text { and } \mathfrak{B}_{\phi}\left(\left\{V_{i}\right\}\right),
$$


while $\left(\boldsymbol{v}_{a, \boldsymbol{z}}, \psi_{a, \boldsymbol{z}}\right)$ denotes the perturbation induced by the nucleating infinitesimal obstacle, such that

$$
\left(\boldsymbol{v}_{a, \boldsymbol{z}}, \psi_{a, \boldsymbol{z}}\right) \text { solves } \quad \mathfrak{E}\left(\Omega_{a, \boldsymbol{z}}\right), \mathfrak{I}_{\mathfrak{u}}, \mathfrak{I}_{\phi}, \mathfrak{B}_{\mathfrak{e r t}}, \mathfrak{B}_{\phi}(0) \text { and } \mathfrak{B}_{\mathfrak{u}}\left(\partial D_{a, \boldsymbol{z}},-\mathcal{C}: \varepsilon[\boldsymbol{u}] \cdot \boldsymbol{n}\right)
$$

Following earlier works on topological sensitivity, e.g. [45, 21, 12], one seeks the asymptotic behavior of $\mathbb{J}\left(\varphi_{a, z} ; T_{f}\right)$ as $a \rightarrow 0$ through an expansion of the cost functional (23) about $\left(\boldsymbol{u}_{a, \boldsymbol{z}}, \varphi_{a, \boldsymbol{z}}\right)=\left(\boldsymbol{u}_{\mid \Omega_{a, \boldsymbol{z}}}, \varphi_{\mid \Omega_{a, \boldsymbol{z}}}\right)$ to first order w.r.t. $\left(\boldsymbol{v}_{a, \boldsymbol{z}}, \psi_{a, \boldsymbol{z}}\right)=\left(\boldsymbol{u}_{a, \boldsymbol{z}}-\boldsymbol{u}_{\mid \Omega_{a, \boldsymbol{z}}}, \varphi_{a, \boldsymbol{z}}-\varphi_{\mid \Omega_{a, \boldsymbol{z}}}\right)$, i.e.

$$
\mathbb{J}\left(\varphi_{a, \boldsymbol{z}} ; T_{f}\right) \underset{a \rightarrow 0}{=} \mathbb{J}\left(\varphi ; T_{f}\right)+\left[\mathbb{J}^{\prime}\left(\varphi ; T_{f}\right)\right]\left(\psi_{a, \boldsymbol{z}}\right)+o\left(\left\|\psi_{a, \boldsymbol{z}}\right\|\right)
$$

where $\lim _{a \rightarrow 0}\left\|\psi_{a, \boldsymbol{z}}\right\|=0$ and $\mathbb{J}^{\prime}$ is the derivative of $\mathbb{J}$ in the direction of the field perturbation.

Owing to first-order Taylor expansion of the misfit function, one has

$$
\left[\mathbb{J}^{\prime}\left(\varphi ; T_{f}\right)\right]\left(\psi_{a, \boldsymbol{z}}\right)=\int_{0}^{T_{f}} \sum_{i=1}^{N_{B}}\left(\varphi-\varphi^{\mathrm{obs}}\right)_{\mid \Gamma_{i}^{c}} \psi_{a, \boldsymbol{z} \mid \Gamma_{i}^{c}} \mathrm{~d} t .
$$

Evaluating (27) is done by quantifying the leading asymptotic behavior in asymptotics (26) as $a \rightarrow 0$. One possible way, along the lines of the so-called direct differentiation approach of parameter or shape sensitivity analysis [23], consists in seeking the asymptotic behavior of $\psi_{a, z}$ on $\Gamma_{i}^{c}$ for $i \in\left\{1, \ldots, N_{B}\right\}$ and plugging the result into (27). As previously discussed on several occasions [10, 6], however, a more compact formulation for actual evaluation of $\left[\mathbb{J}^{\prime}\left(\varphi_{\mid \Omega_{a, z}}\right)\right]\left(\psi_{a, z}\right)$ can be set up using an adjoint-field based approach and is adopted here.

\subsection{Piezoelectric sensor model}

\subsubsection{Adjoint-field formulation}

The key idea of the adjoint formulation stems from treating the integral in the right-hand side of (27) as one of the terms arising in the reciprocity identity (15) linking two piezoelectric states, in which one state is the perturbation $\left(\boldsymbol{v}_{a, \boldsymbol{z}}, \psi_{a, \boldsymbol{z}}\right)$ while the other is chosen as a so-called adjoint solution $(\hat{\boldsymbol{u}}, \hat{\varphi})$ to be defined appropriately.

Consider the electric inputs $\left\{\hat{V}_{i}\right\}$ defined by the time-reversed counterpart of the measurements residuals, i.e.

$$
\hat{V}_{i}(t)=\left(\varphi-\varphi^{\mathrm{obs}}\right)_{\mid \Gamma_{i}^{c}}\left(T_{f}-t\right) \quad \text { for } i \in\left\{1, \ldots, N_{B}\right\}, t \in\left[0, T_{f}\right]
$$

and let $(\hat{\boldsymbol{u}}, \hat{\varphi})$ be a piezoelectric solution in the reference defect-free domain $\Omega$ of the following problem

$$
(\hat{\boldsymbol{u}}, \hat{\varphi}) \quad \text { solves } \mathfrak{E}(\Omega), \mathfrak{I}_{\mathfrak{u}}, \mathfrak{I}_{\phi}, \mathfrak{B}_{\mathfrak{e x t}} \text { and } \mathfrak{B}_{\phi}\left(\left\{\hat{V}_{i}\right\}\right)
$$

Now, on applying Corollary 4.1 to the solutions $(\hat{\boldsymbol{u}}, \hat{\varphi})$ and $\left(\boldsymbol{v}_{a, \boldsymbol{z}}, \psi_{a, \boldsymbol{z}}\right)$ in the subdomain $\mathcal{O}=\Omega_{a, \boldsymbol{z}}$ and owing to the corresponding initial conditions and applied excitations in $(25,29)$, then equation (15) is recast in

$$
R \frac{d}{d t} \int_{\partial \Omega_{a, \boldsymbol{z}}}\left\{(\mathcal{C}: \varepsilon[\hat{\boldsymbol{u}}]+\mathbf{d} \cdot \boldsymbol{\nabla} \hat{\varphi}) \cdot \boldsymbol{n}_{\underline{\star}} \boldsymbol{v}_{a, \boldsymbol{z}}-\left(\mathcal{C}: \varepsilon\left[\boldsymbol{v}_{a, \boldsymbol{z}}\right]+\mathbf{d} \cdot \boldsymbol{\nabla} \psi_{a, \boldsymbol{z}}\right) \cdot \boldsymbol{n} \underset{\underline{\boldsymbol{u}}}{ }\right\} \mathrm{d} \sigma=\sum_{i=1}^{N_{B}} \hat{V}_{i} \star \psi_{a, \boldsymbol{z} \mid \Gamma_{i}^{c}} .
$$


On using definition (28), then the right-hand sides of the equations (27) and (30) turn out to be equal, therefore, and owing to hypothesis $(3)$ and the relevant boundary conditions in $(25,29)$ on the infinitesimal defect boundary $\partial D_{a, z}$ as well as on the exterior surface $\partial \Omega_{a, \boldsymbol{z}} \backslash \partial D_{a, \boldsymbol{z}}$, the derivative $\mathbb{J}^{\prime}$ reduces to

$$
\left[\mathbb{J}^{\prime}\left(\varphi ; T_{f}\right)\right]\left(\psi_{a, \boldsymbol{z}}\right)=\sum_{i=1}^{N_{B}} \hat{V}_{i} \star \psi_{a, \boldsymbol{z} \mid \Gamma_{i}^{c}}=R \frac{d}{d t} \int_{\partial D_{a, \boldsymbol{z}}}\left\{\mathcal{C}: \boldsymbol{\varepsilon}[\boldsymbol{u}] \cdot \boldsymbol{n}_{\star} \hat{\boldsymbol{u}}+\mathcal{C}: \varepsilon[\hat{\boldsymbol{u}}] \cdot \boldsymbol{n}_{\star} \boldsymbol{v}_{a, \boldsymbol{z}}\right\} \mathrm{d} \sigma .
$$

\subsubsection{Topological derivative}

Next, the so-called topological derivative function $\mathbb{T}$ can be defined through the identity

$$
\left[\mathbb{J}^{\prime}\left(\varphi ; T_{f}\right)\right]\left(\psi_{a, \boldsymbol{z}}\right) \underset{a \rightarrow 0}{=} \eta(a) \mathbb{T}\left(\boldsymbol{z} ; \mathcal{D}, T_{f}\right)+o(\eta(a)),
$$

where the function $\eta(a)$ vanishing in the limit $a \rightarrow 0$. The coupled piezoelectric state $\left(\boldsymbol{v}_{a, \boldsymbol{z}}, \psi_{a, \boldsymbol{z}}\right)$ exhibits an asymptotical behavior, however, as emphasized in the equation (31), one is only interested in the leading contribution of $\boldsymbol{v}_{a, \boldsymbol{z}}$ on $\partial D_{a, z}$ as $a \rightarrow 0$. Since the sampling point $\boldsymbol{z}$ varies within the elastic domain $\Omega_{S}$, i.e. not within the piezoelectric material domain $\Omega_{T}$, then in the limit $a \rightarrow 0$ the coupled boundary condition at the transducer interface $\partial \Omega_{T} \cap \partial \Omega_{S}$ does not enter the asymptotics of $\boldsymbol{v}_{a, \boldsymbol{z}}$. Consequently, the expressions established by local analysis for purely elastic configurations in, e.g., $[45,21,22,3]$, remain valid in the present situation and hence, the right-hand side of equation (31) can now be computed by inserting the appropriate asymptotic behavior of $\boldsymbol{v}_{a, \boldsymbol{z}}$, see details provided in Section 3 of

[6]. Finally, with the second-order stress tensor defined as $\sigma[\boldsymbol{v}]=\mathcal{C}: \varepsilon[\boldsymbol{v}]$ in $\mathcal{L}\left(\mathbb{R}^{d}\right)$ and the symbol $\underline{=}$ denoting a time convolution involving the inner product (2), one obtains

$$
\begin{aligned}
& \eta(a)=a^{d}|\mathcal{D}|, \\
& \mathbb{T}\left(\boldsymbol{z} ; \mathcal{D}, T_{f}\right)=R \frac{d}{d t}\left(\boldsymbol{\sigma}[\boldsymbol{u}]_{\underline{\underline{ }}} \mathcal{A}(\mathcal{D}): \boldsymbol{\sigma}[\hat{\boldsymbol{u}}]+\beta \frac{d \boldsymbol{u}}{d t} \star \frac{d \hat{\boldsymbol{u}}}{d t}\right)(\boldsymbol{z}),
\end{aligned}
$$

where the so-called elastic moment tensor $\mathcal{A} \in \mathcal{L}^{2}\left(\mathbb{R}^{d}\right)$ depends on the shape of the infinitesimal traction-free obstacle $D_{a, z}$ that is featured in the asymptotic analysis, while regardless of this cavity shape $\beta=\rho$ (see, e.g., [22]).

The computation of the indicator function $\mathbb{T}$ requires the knowledge of the free-field $\boldsymbol{u}$ and the adjoint-field $\hat{\boldsymbol{u}}$ respectively defined by the problems (24) and (29) in the reference defect-free domain $\Omega$. Moreover, the parameter $\mathcal{A}$ entering the definition (32b) can be computed for any arbitrary shape $\mathcal{D}$ by solving canonical elastostatic exterior problems [22]. However, it can be found in a closed form when the assumed obstacle shape $\mathcal{D}$ in relatively simple, e.g. $\mathcal{D}$ being a sphere in $\mathbb{R}^{3}$. Such a closed form is preferred for the purposes of the present study, and the formula established in previous studies are listed hereafter for the reader's convenience.

Consider the case where $\Omega_{S}$ is a homogeneous linear elastic and isotropic solid characterized by shear modulus $\mu$ and Poisson's ratio $\nu$, so that the elasticity tensor reduces to

$$
\mathcal{C}=2 \mu\left[\mathcal{I}^{\text {sym }}+\frac{\nu}{1-2 \nu} \boldsymbol{I} \otimes \boldsymbol{I}\right]
$$


where $\mathcal{I}^{\text {sym }}$ and $\boldsymbol{I}$ denote respectively the symmetric fourth-order and second-order identity tensors. Then, for the configurations addressed in this study, the material parameters in (32b) reduce to the following expressions depending on the chosen shape $\mathcal{D}$ : For the case of a spherical (resp. circular) cavity nucleating in the 3D (resp. 2D) elastic body considered, one has $|\mathcal{D}|=4 \pi / 3$ (3D) or $|\mathcal{D}|=\pi$ (2D) and from [21]

$$
\begin{aligned}
& \mathcal{A}=\frac{3(1-\nu)}{2 \mu(7-\nu)}\left[5 \mathcal{I}^{\text {sym }}-\frac{1+5 \nu}{2(1+\nu)} \boldsymbol{I} \otimes \boldsymbol{I}\right] \\
& \mathcal{A}=\frac{1-\nu}{\mu}\left[2 \mathcal{I}^{\text {sym }}-\frac{1}{2(1+\nu)} \boldsymbol{I} \otimes \boldsymbol{I}\right]
\end{aligned}
$$

Remark 6.1 Note that the previous developments are not intrinsically limited to a choice of elastic boundary conditions such as (8) and the proposed approach can be easily generalized to the case of inhomogeneous elastic scatterers. In such a case, the parameters $\mathcal{A}$ and $\beta$ depend additionally on the assumed elastic parameters of the infinitesimal trial obstacle. In the case of a small nucleating spherical elastic inclusion, closed-form expressions can be found in [16]. Further generalizations of the topological sensitivity approach include the case of traction-free cracks [7]

\section{Numerical results}

\subsection{Implementation}

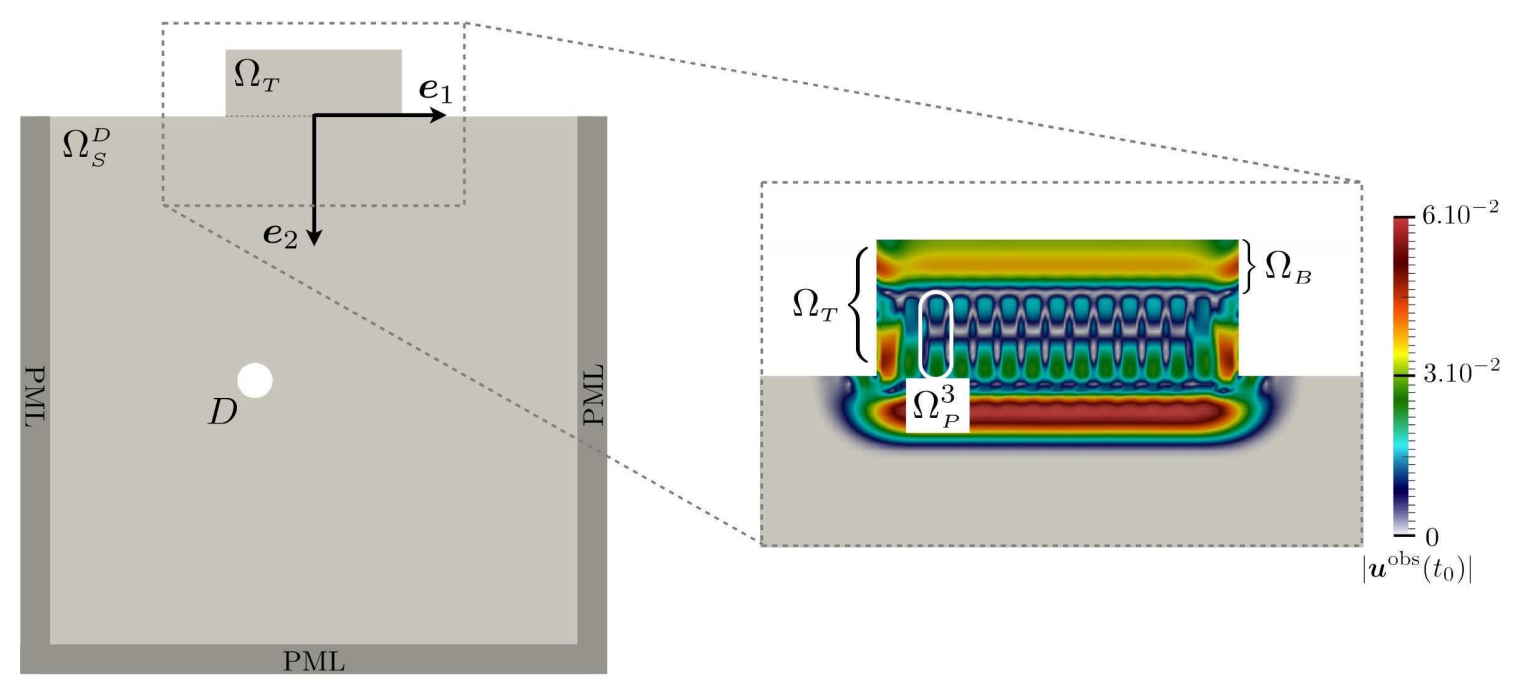

Figure 2: Left: Schematic of $2 D$ configuration $\Omega_{D}$. Right: Zoom on transducer characterized by $N_{B}=15$, delineated piezoelectric bar $\Omega_{P}^{3}$ and amplitude of total displacement field $\boldsymbol{u}^{\mathrm{obs}}$ in $\mathrm{nm}$ at time $t_{0}=23 \mu \mathrm{s}$.

Typical configurations in the field of non-destructive testing are not unbounded, however piezoelectric transducers are commonly employed to investigate locally such structures, i.e. within areas that are small with respect to the size 
of the overall body which can then be seen as unbounded at the scale considered. Therefore, the numerical experiments presented hereafter correspond to the probing of a two-dimensional and homogeneous half-space $\Omega_{S}^{D}$. An example geometry of a studied configuration is described Figure 2 with width and height corresponding respectively to dimensions along axes $e_{1}$ and $e_{2}$ : The transducer of width $l=30 \mathrm{~mm}$ is composed of $N_{B}$ equidistributed bars of width $l / 2 N_{B}$ and height $6.3 \mathrm{~mm}$. A $5 \mathrm{~mm}$-height dissipative backing denoted $\Omega_{B}$ lies atop the domain $\Omega_{P}$, so that the dissipation parameter $\alpha$ in equation (4) is characterized by $\operatorname{supp} \alpha=\Omega_{B}$. Finally, the inter-bar space $\Omega_{T} \backslash\left(\Omega_{B} \cup \Omega_{P}\right)$ is considered to be filled with a soft isotropic elastic matrix. Chosen physical parameters are summarized in Table 1 and correspond to a standard PZT piezoelectric material [40]. Note also, that common 2D transducer configurations involve a number $N_{B}$ of active elements of the order of a dozen [41]. On Fig. 2, the unknown defect $D$ embedded in the isotropic elastic medium $\Omega_{S}$ is a circular cavity, with radius $r=3 \mathrm{~mm}$ and center $c=(-10 \mathrm{~mm}, 45 \mathrm{~mm})$.

The spatial discretization relies on the spectral finite-elements method (see [17] and [28] for more details), and featured elements are associated with 6 -th order polynomial basis functions. The medium $\Omega_{S}^{D}$ is truncated using surrounding Perfectly Matched Layers (PML) [18], to obtain a computational domain of size $90 \mathrm{~mm} \times 90 \mathrm{~mm}$ which is discretized using 52417 nodes. The discretized transducer $\Omega_{T}$ and PML domains are respectively associated with 10544 and 17525 nodes. The different sub-meshes composing the global mesh are coupled by a standard non-conforming/non-overlapping mortar element technique [9]. As in [27], the time discretization is done via an explicit second-order energy-preserving finite-difference scheme and the stability of the fully discrete problem is guaranteed through an energy approach under a standard CFL condition. For the simulations the time step is set to $\Delta t=0.01 \mu$ s while $T_{f}=200 \mu$ s. Note that in order to limit the memory requirements then the time convolutions associated with the computation of (32b) are discretized using a time step equals to $10 \Delta t$.

Figure 3 represents snapshots of incident field $\boldsymbol{u}$, simulated observation field $\boldsymbol{u}^{\text {obs }}$ and corresponding adjoint field $\hat{\boldsymbol{u}}$. In this experiment each piezoelectric bar cathode $\Gamma_{i}^{c}$ is connected to a generator which resistance is set to $R=400$

\begin{tabular}{|c|c|c|c|c|c|c|c|c|}
\hline \multicolumn{9}{|c|}{ Piezoelectric bar $\Omega_{P}^{i}$} \\
\hline $\mathcal{C}_{1111}$ & 111.0 & $\mathcal{C}_{1122}$ & 15.4 & $\mathcal{C}_{2222}$ & $\mathcal{C}_{1212}$ & 21.1 & $\rho$ & $7.75 \times 10^{3}$ \\
\hline $\mathrm{d}_{112}$ & 12.7 & $\mathrm{~d}_{211}$ & -5.4 & $\mathrm{~d}_{222}$ & 15.1 & 1730 & $\epsilon_{22}$ & 1700 \\
\hline \multicolumn{9}{|c|}{ Isotropic elastic matrix $\Omega_{T} \backslash\left(\Omega_{B} \cup \Omega_{P}\right)$} \\
\hline $\mathcal{C}_{1111}$ & 8.5 & $\mathcal{C}_{1122}$ & 4.3 & $\rho$ & $1.1 \times 10^{3}$ & & & \\
\hline \multicolumn{9}{|c|}{ Isotropic and dissipative backing $\Omega_{B}$} \\
\hline $\mathcal{C}_{1111}$ & 14.4 & $\mathcal{C}_{1122}$ & 7.22 & $\rho$ & $1.8 \times 10^{3}$ & $1.8 \times 10^{-5}$ & & \\
\hline \multicolumn{9}{|c|}{ Isotropic elastic medium $\Omega_{S}$} \\
\hline $\mathcal{C}_{1111}$ & 37.15 & $\mathcal{C}_{1122}$ & 10.88 & $\rho$ & $6.8 \times 10^{3}$ & & & \\
\hline
\end{tabular}

Table 1: Electric and elastic material parameters. Units reads $\left[\mathcal{C}_{i j k l}\right]=\mathrm{GPa},[\rho]=\mathrm{kg} \cdot \mathrm{m}^{-3},\left[\mathrm{~d}_{i j k}\right]=\mathrm{C} \cdot \mathrm{m}^{-2}$, $\epsilon_{i j}$ given relatively to vacuum permittivity and scalar parameter $\alpha$. 

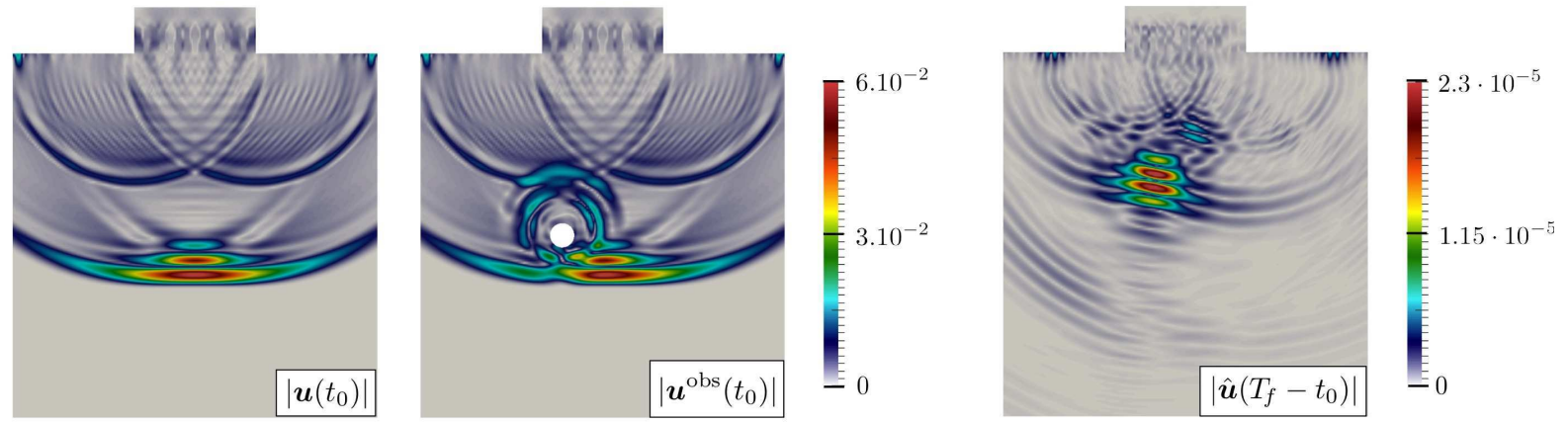

Figure 3: Amplitude of computed fields at time $t_{0}=45 \mu$ s during a numerical experiment with $N_{B}=15$ : Incident field $\boldsymbol{u}$, observation $\boldsymbol{u}^{\mathrm{obs}}$ and associated adjoint field $\hat{\boldsymbol{u}}$ in $\mathrm{nm}$.

Ohm. To compute the incident displacement field $\boldsymbol{u}$ and generate the synthetic observations $\boldsymbol{u}^{\text {obs }}$, it is assumed that all cathodes are excited using the common source function

$$
V_{i}\left(t ; \tau, f_{0}\right)= \begin{cases}{\left[1-\cos \left(2 \pi f_{0}(t-\tau)\right)\right] \text { when } t \in\left[\tau, 1 / f_{0}+\tau\right]} & \text { for } i=1, \ldots, N_{B} \\ 0 \text { otherwise } & \end{cases}
$$

with $f_{0}=600 \mathrm{kHz}$ and $\tau=20 \mu \mathrm{s}$. electric input and corresponding measurements on three different electrodes are plotted Figure 4.

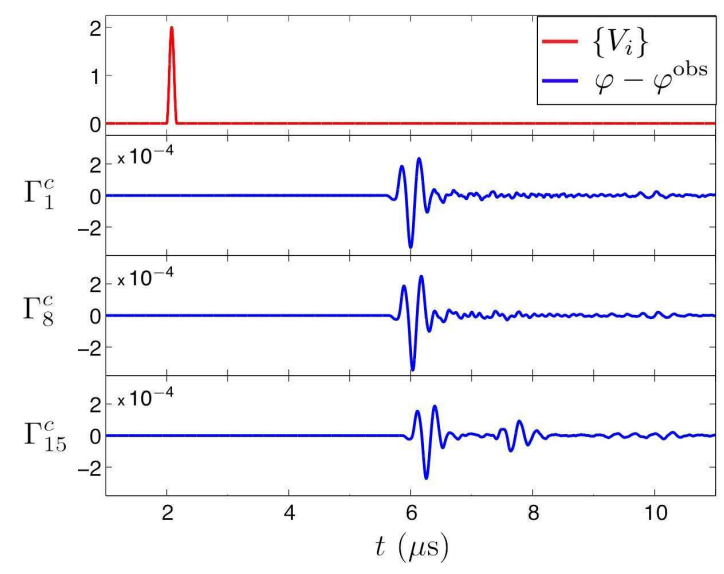

Figure 4: Electric potentials in $\mathrm{V}$ corresponding to experiment of Fig. 3: electric inputs $V_{i}$ with $i=$ $1, \ldots, N_{B}$ and measured residual potentials $\varphi-\varphi^{\text {obs }}$ on three different electrodes.

\subsection{Dirichlet-type vs Piezoelectric measurements}

To illustrate the issues raised in Section 5.2, comparisons are provided in this section between computed elastodynamic solutions in the defect-free medium $\Omega_{S}$ for configurations involving or not coupling with the piezoelectric transducer. 


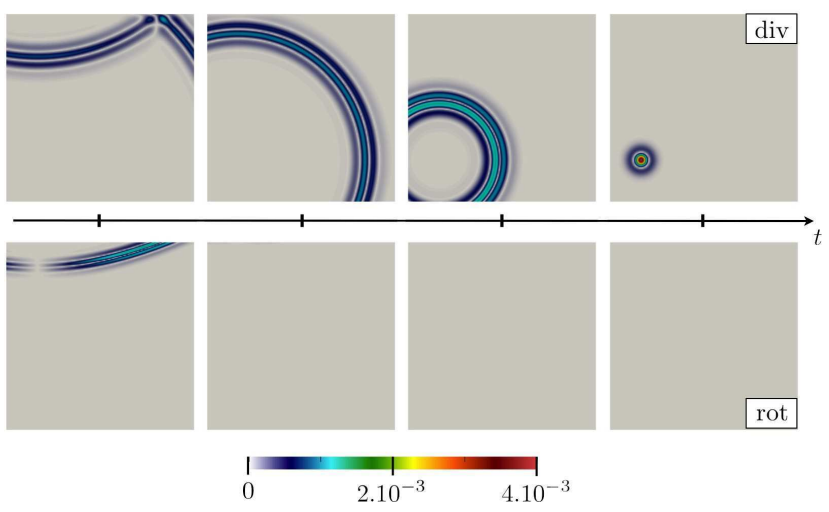

(a) Free-surface b.c.: Time-reversed incident field $\boldsymbol{u}\left(T_{f}-t\right)$

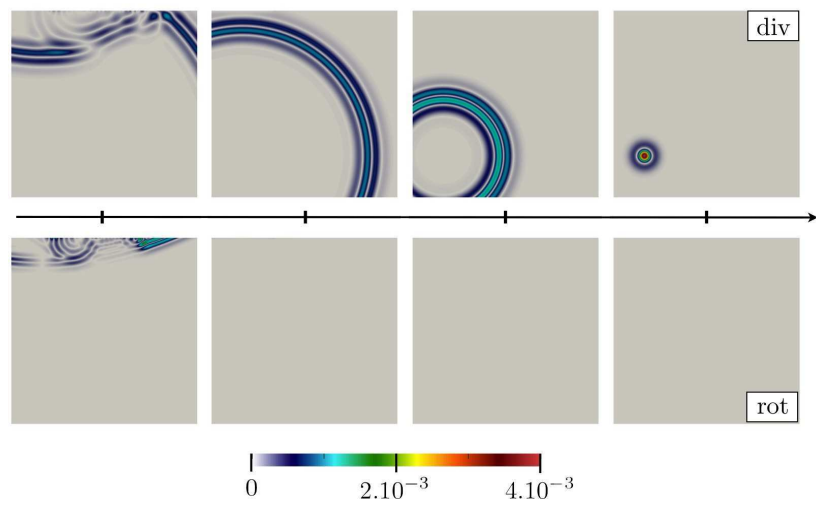

(c) Piezoelectric b.c.: Time-reversed incident field $\boldsymbol{u}\left(T_{f}-t\right)$

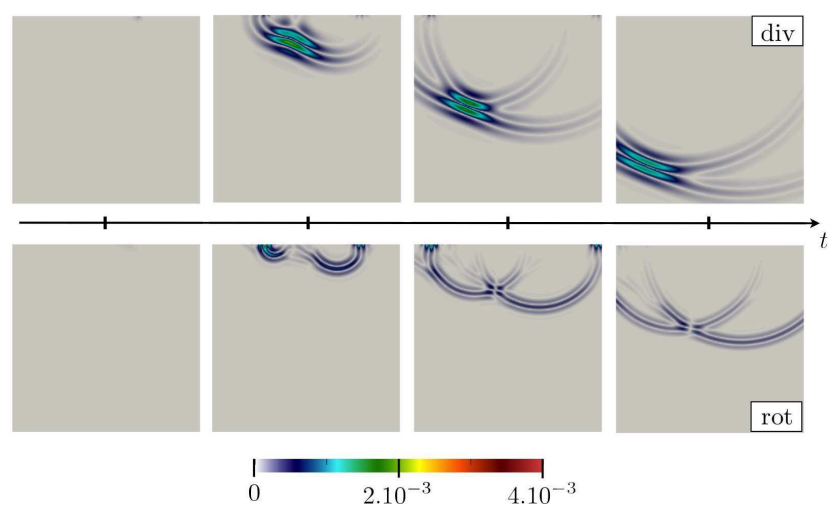

(b) Dirichlet-type measurements: Adjoint field $\hat{\boldsymbol{u}}(t)$
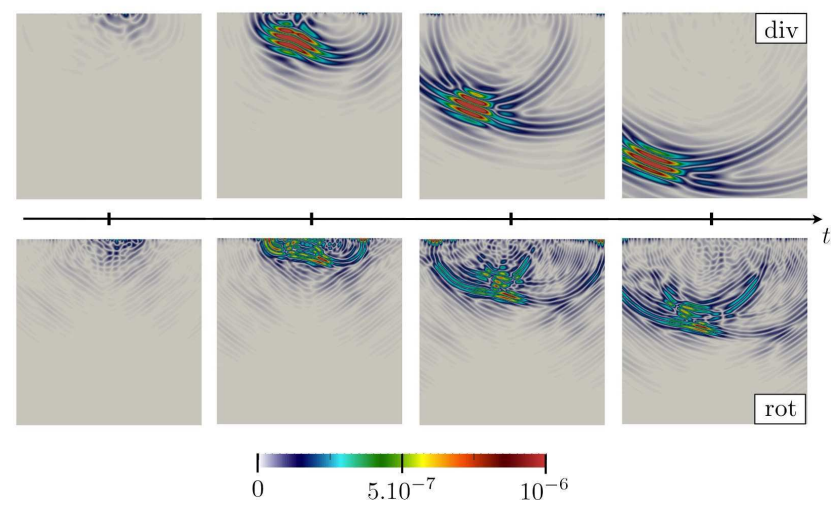

(d) Piezoelectric measurements: Adjoint field $\hat{\boldsymbol{u}}(t)$

Figure 5: P-wave source: Displacements fields in linear isotropic background medium $\Omega_{S}$.

In the different experiments, an elastic source is placed at point $\boldsymbol{x}_{s}=(-35 \mathrm{~mm}, 70 \mathrm{~mm})$ and it is calibrated so as to generate purely divergence- or rotational-free forward elastic solution $\boldsymbol{u}$ up to interaction with the top boundary of the domain considered. The employed body-force terms, denoted $f_{P}$ and $f_{S}$, are defined as

$$
\boldsymbol{f}_{P / S}(\boldsymbol{x}, t)=\boldsymbol{F}_{P / S} e^{-a\left\|\boldsymbol{x}-\boldsymbol{x}_{s}\right\|^{2}} r\left(t ; f_{s}, 0\right) \quad \text { with } r\left(t ; f_{s}, t_{s}\right)=\left[2 \pi^{2}\left(f_{s}\left(t-t_{s}\right)-1\right)^{2}\right] e^{-\pi^{2}\left(f_{s}\left(t-t_{s}\right)-1\right)^{2}},
$$

where the polarized amplitudes read

$$
\boldsymbol{F}_{P}=\frac{\boldsymbol{x}-\boldsymbol{x}_{s}}{\left\|\boldsymbol{x}-\boldsymbol{x}_{s}\right\|+b} \quad \text { and } \quad \boldsymbol{F}_{S}=\frac{\left[\left(\boldsymbol{x}-\boldsymbol{x}_{s}\right) \cdot \boldsymbol{e}_{1}\right] \boldsymbol{e}_{2}-\left[\left(\boldsymbol{x}-\boldsymbol{x}_{s}\right) \cdot \boldsymbol{e}_{2}\right] \boldsymbol{e}_{1}}{\left\|\boldsymbol{x}-\boldsymbol{x}_{s}\right\|+b}
$$

in terms of adjustable parameters set to $f_{s}=200 \mathrm{kHz}, a=8 \cdot 10^{-2} \mathrm{~mm}^{-2}$ and $b=10^{-8} \mathrm{~mm}$. On the one hand, purely elastic simulations are presented as references: Forward solutions $\boldsymbol{u}$ solving the elastodynamic equation in the halfspace $\Omega_{S}$ are computed assuming traction-free boundary conditions on $\partial \Omega_{S}$. Then, full-field boundary measurements of $\boldsymbol{u}$ at 73 nodes along the corresponding subset $\partial \Omega_{T} \cap \partial \Omega_{S}$ of the free-surface are used to generate the adjoint solutions $\hat{\boldsymbol{u}}$. On the other hand, the coupled piezoelectric problem is considered: Forward solutions $(\boldsymbol{u}, \varphi)$ are computed in the presence of a passive sensor, i.e. assuming (24) with electric condition $\mathfrak{B}_{\phi}(0)$, together with corresponding adjoint fields $\hat{\boldsymbol{u}}$ solving (29) with sources $\hat{V}_{i}(t)=\varphi_{\mid \Gamma_{i}^{c}}\left(T_{f}-t\right)$ for $i=1, \ldots, 15$. 


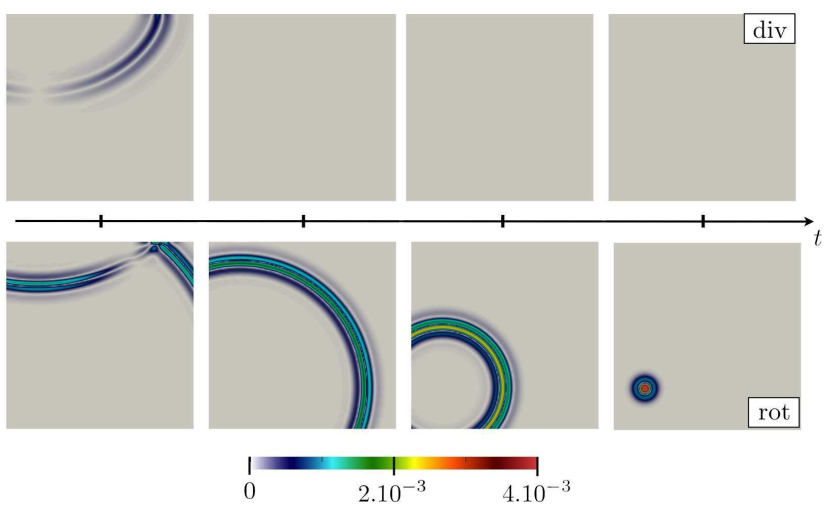

(a) Free-surface b.c.: Time-reversed incident field $\boldsymbol{u}\left(T_{f}-t\right)$

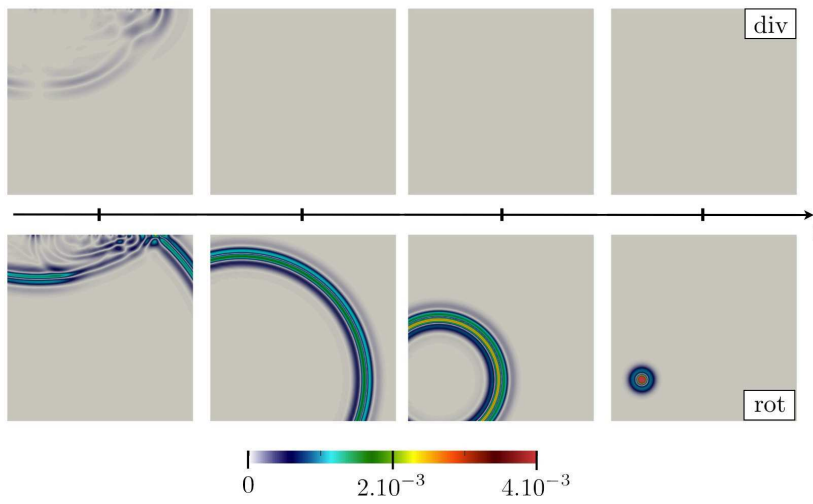

(c) Piezoelectric b.c.: Time-reversed incident field $\boldsymbol{u}\left(T_{f}-t\right)$

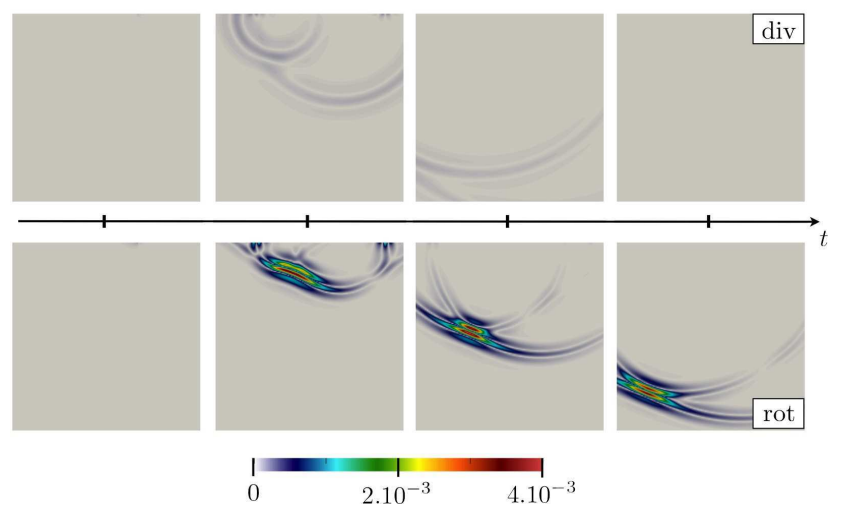

(b) Dirichlet-type measurements: Adjoint field $\hat{\boldsymbol{u}}(t)$

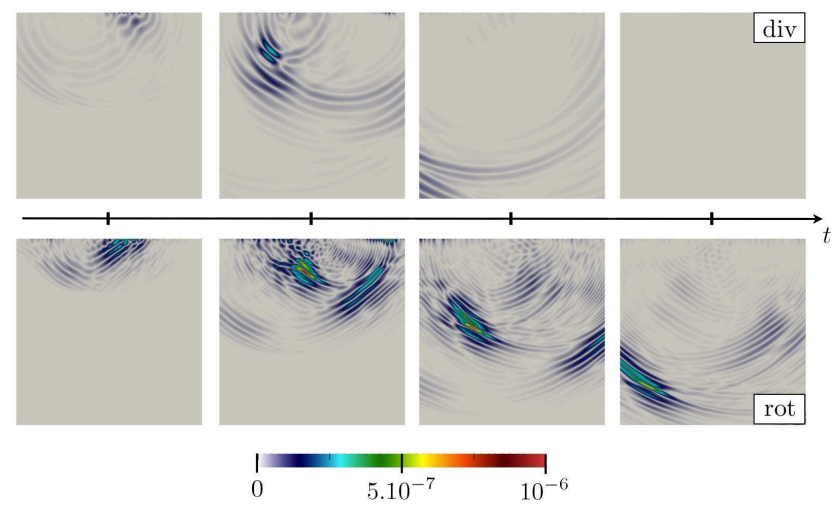

(d) Piezoelectric measurements: Adjoint field $\hat{\boldsymbol{u}}(t)$

Figure 6: $S$-wave source: Displacements fields in linear isotropic background medium $\Omega_{S}$.

Figures 5 and 6 represent snapshots of the computed solutions at four equidistributed instants in $] 0, T[$. Each field is decomposed into divergence (resp. rotational) component shown in the top (resp. bottom) panels. To facilitate the comparison between them, time-reversed counterparts of forward solutions $\boldsymbol{u}$ are plotted aside forward representations of corresponding adjoint states $\hat{\boldsymbol{u}}$.

For the two types of excitation considered on figures 5 and 6, comparison between Fig. a and b, as well as Fig. c and d, highlights the analogies between $\boldsymbol{u}\left(T_{f}-t\right)$ and $\hat{\boldsymbol{u}}(t)$ : The adjoint field is predominantly of the same nature, in terms of amplitude of divergence or rotational component, than the original forward solution. Moreover, the solutions $\hat{u}$ achieve focusing in the area of the original source location. Now, comparing Fig. 5b and 5d, or Fig. 6b and 6d, emphasizes significant differences between full-field measurements based and piezoelectric adjoint states. In the case of the P-wave source (resp. S-wave) and piezoelectric measurements, the computed adjoint field exhibits a rotational (resp. divergence) component of significantly higher relative amplitude. Moreover, the wave-fronts appear to be relatively distorted on Figures $5 \mathrm{~d}$ and $6 \mathrm{~d}$, a phenomenon which can be accounted for by the complexity induced by the coupling with the piezoelectric sensor. 


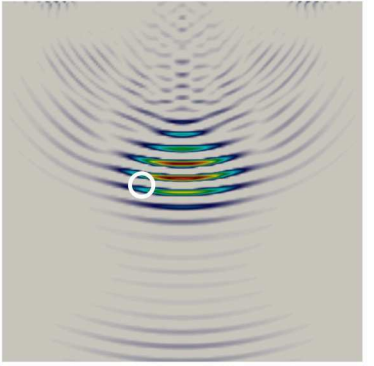

(a) $N_{B}=1$

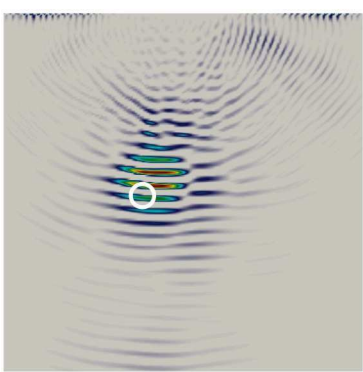

(d) $N_{B}=9$

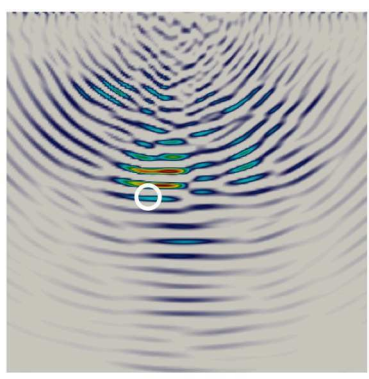

(b) $N_{B}=3$

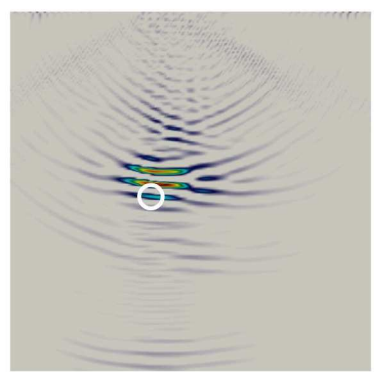

(e) $N_{B}=15$

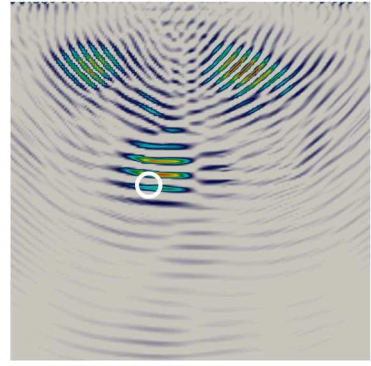

(c) $N_{B}=5$

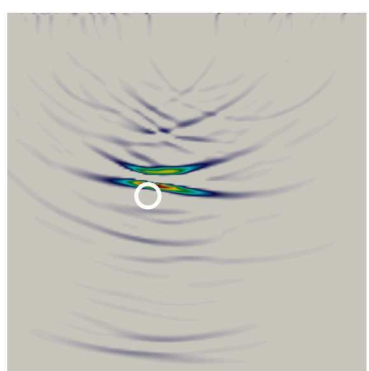

(f) Ref.

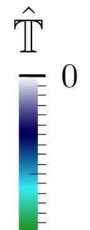

$-0.5$

-1

Figure 7: Identification of a single cavity: Increasing number $N_{B}$ of piezoelectric bars (Fig. a-e) and comparison with result $\mathrm{f}$ based on direct boundary measurement of displacement field.

\subsection{Topological sensitivity based identification}

Heuristic. Identification using the topological sensitivity based defect indicator function is addressed in this section. The topological derivative $\mathbb{T}\left(\boldsymbol{z} ; \mathcal{D}, T_{f}\right)$ quantifies the sensitivity of the cost functional $\mathbb{J}$ considered to the nucleation at point $\boldsymbol{z}$ of an infinitesimal trial cavity in the reference medium $\Omega_{S}$. Therefore, the function $\boldsymbol{z} \mapsto \mathbb{T}\left(\boldsymbol{z} ; \mathcal{D}, T_{f}\right)$ is employed as a cavity indicator function with the heuristic approach of seeking actual $\operatorname{defect}(\mathrm{s})$ at locations $z$ at which $\mathbb{T}\left(\boldsymbol{z} ; \mathcal{D}, T_{f}\right)$ attains its most pronounced negative values (i.e. such that infinitesimal trial cavity placed there improve the fit between predicted and actual measurements). While intuition (and previous studies on the same approach carried out for other types of defects $[22,16,6])$ suggests that finite defects having the same location also induce a decrease of the cost function, this proposed exploitation of the field $\mathbb{T}\left(\cdot ; \mathcal{D}, T_{f}\right)$ is not backed by a rigorous mathematical proof (whereas the analysis of the cost function leading to the definition and evaluation of $\mathbb{T}\left(\boldsymbol{z} ; \mathcal{D}, T_{f}\right)$ is itself mathematically rigorous). This proposed heuristic identification approach will be tested on numerical experiments in this section.

The indicator function (32b) featuring polarization tensor (33b) corresponding to the $2 \mathrm{D}$ plane strain model is computed and a thresholded and normalized version $\hat{\mathbb{T}}$ is defined as

$$
\hat{\mathbb{T}}\left(\boldsymbol{z} ; \mathcal{D}, T_{f}\right)= \begin{cases}\mathbb{T}\left(\boldsymbol{z} ; \mathcal{D}, T_{f}\right) /\left|\min _{\boldsymbol{x} \in \Omega_{S}} \mathbb{T}\left(\boldsymbol{x} ; \mathcal{D}, T_{f}\right)\right| & \text { if } \mathbb{T}\left(\boldsymbol{z} ; \mathcal{D}, T_{f}\right)<0 \\ 0 & \text { if } \mathbb{T}\left(\boldsymbol{z} ; \mathcal{D}, T_{f}\right)>0\end{cases}
$$




\subsubsection{Single cavity identification}

The identification of a single circular cavity of radius $r=3 \mathrm{~mm}$ is considered here with its center located at $c_{1}=$ $(-10 \mathrm{~mm}, 45 \mathrm{~mm})$ (Fig. 7) or $c_{2}=(-30 \mathrm{~mm}, 45 \mathrm{~mm})$ (Fig. 8). Panels a-e show the reconstructions obtained using piezoelectric measurements and an increasing number $N_{B}$ of piezoelectric bars that are commonly excited using the source function (34). As references, results obtained in purely elastic configurations, i.e. in the absence of piezoelectric transducer, are also included on panels $\mathrm{f}$. These reconstructions follow the methodology described in [6]: Data $\boldsymbol{u}^{\text {obs }}$ correspond to simulations where transient excitations are imposed in the form of the following applied tractions

$$
\mathcal{C}: \varepsilon\left[\boldsymbol{u}^{\mathrm{obs}}\right] \cdot \boldsymbol{n}=r\left(t ; f_{s}, t_{s}\right) \chi_{\partial \Omega_{S}} \cup \partial \Omega_{T} \boldsymbol{e}_{2} \quad \text { on } \partial \Omega_{S}, \text { with } f_{s}=3 \cdot 10^{5} \mathrm{~Hz} \text { and } t_{s}=10 \mu \mathrm{s},
$$

with characteristic function $\chi_{\partial \Omega_{S}} \cup \partial \Omega_{T}$, and recording full-field measurements at 73 nodes along the corresponding part $\partial \Omega_{S} \cup \partial \Omega_{T}$ of the top boundary.

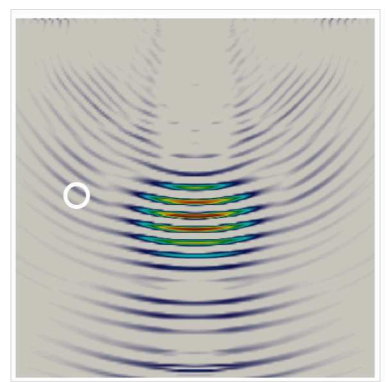

(a) $N_{B}=1$

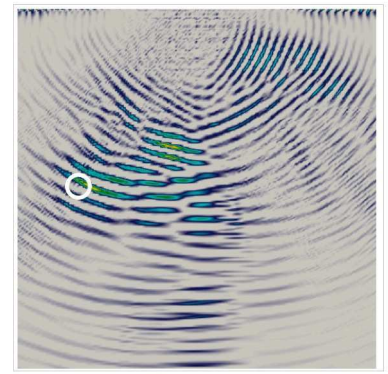

(d) $N_{B}=9$

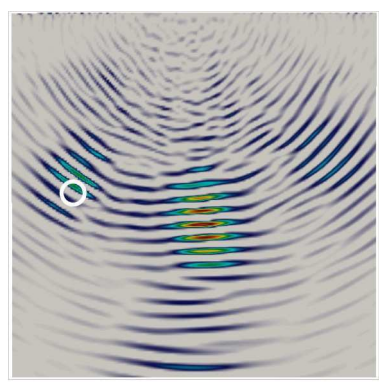

(b) $N_{B}=3$

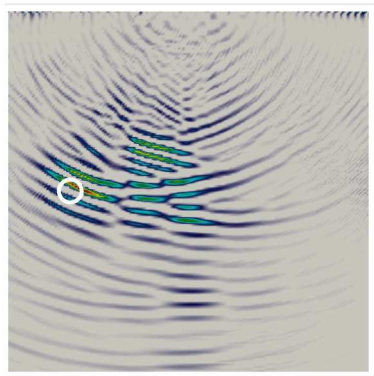

(e) $N_{B}=15$

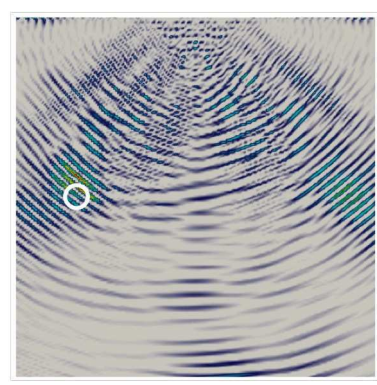

(c) $N_{B}=5$

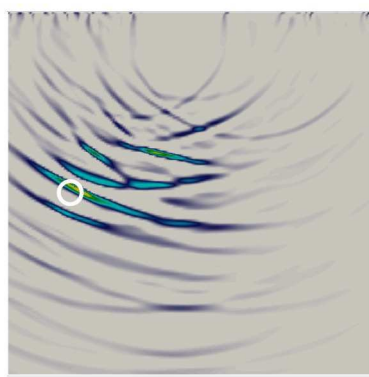

(f) Ref.

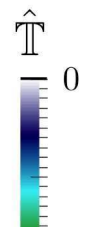

$-0.5$

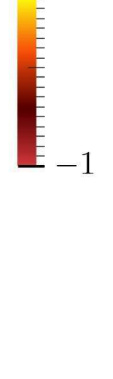

$-1$

Figure 8: Identification of a single cavity: Increasing number $N_{B}$ of piezoelectric bars (Fig. a-e) and comparison with result $\mathrm{f}$ based on direct boundary measurement of displacement field.

Finally, Figure 9a-c show the influence of introducing an additional cutoff of the normalized topological gradient indicator function $\hat{\mathbb{T}}$ which are here respectively associated with values less than $-0.1,-0.2$ and -0.3 .

\subsubsection{Multiple cavity identification using optimally-focusing excitations}

In this section, results based on optimally-focusing excitations are presented for multiple cavity configurations and employing a transducer composed of a number $N_{B}=15$ of piezoelectric bars. Configuration 1 involves two cavities with 


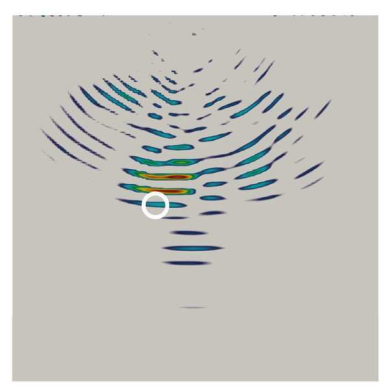

(a)

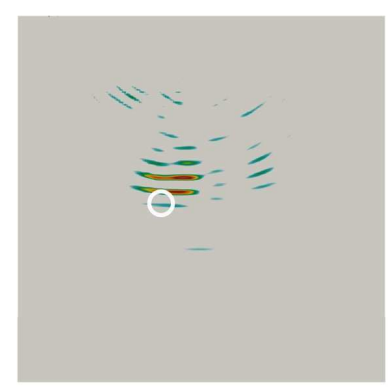

(b)

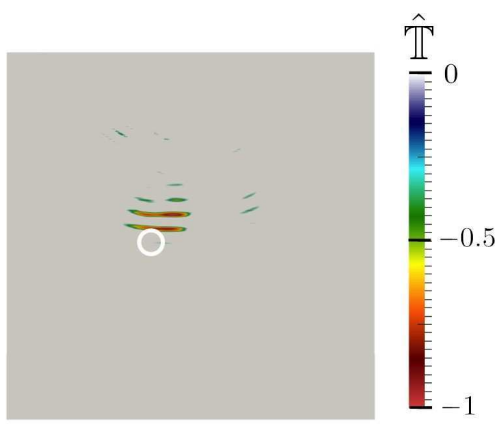

(c)

Figure 9: Configuration of Figure 7b: Comparison for the cutoff values $-0.1,-0.2$ and -0.3 .

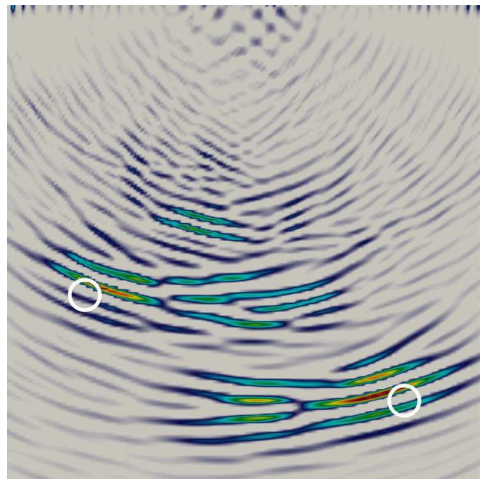

(a) Config. 1: Two cavities

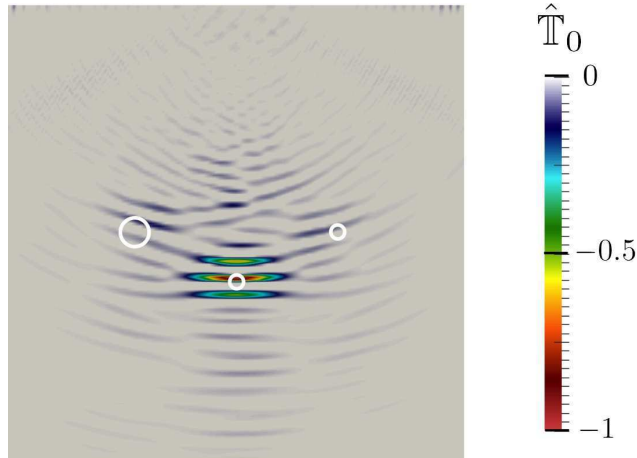

(b) Config. 2: Three cavities

Figure 10: Simultaneous identification of multiple cavities based on $N_{B}=15$ piezoelectric bars.

common radius $r=3 \mathrm{~mm}$ and centers $\boldsymbol{c}_{1}=(-30 \mathrm{~mm}, 55 \mathrm{~mm})$ and $\boldsymbol{c}_{2}=(30 \mathrm{~mm}, 75 \mathrm{~mm})$; Configuration 2 features three cavities located at $\boldsymbol{c}_{1}=(-20 \mathrm{~mm}, 45 \mathrm{~mm}), \boldsymbol{c}_{2}=(0 \mathrm{~mm}, 55 \mathrm{~mm})$ and $\boldsymbol{c}_{3}=(20 \mathrm{~mm}, 45 \mathrm{~mm})$ with corresponding radii $r_{1}=3 \mathrm{~mm}$ and $r_{2}=r_{3}=1.5 \mathrm{~mm}$.

Electric inputs $\left\{V_{i}\right\}_{n}$ are defined iteratively using the power iteration method (19) presented in Section 4.3:

- Let $\left\{V_{i}\right\}_{0} \in L^{2}\left(\left[0, T_{f}\right]\right)^{N_{B}}$ denote the initial electric input defined by (34)

- Compute $\left(\boldsymbol{u}_{n}, \varphi_{n}\right)$ in $\Omega_{T} \cup \Omega_{S}$ and synthetic observation $\left(\boldsymbol{u}_{n}^{\text {obs }}, \varphi_{n}^{\text {obs }}\right)$ in $\Omega_{D}$ associated with input $\left\{V_{i}\right\}_{n}$

- Compute $\left\{W_{i}\right\}_{n+1}=\left[\mathcal{R} \mathcal{S}\left(\mathcal{P}_{D}-\mathcal{P}\right)\right]\left(\left\{V_{i}\right\}_{n}\right)=\left\{\left[s \star\left(\varphi_{n}^{\mathrm{obs}}-\varphi_{n}\right)_{\Gamma_{i}^{c}}\right]\left(T_{f}-t\right)\right\}$

with filter function $s(t)=\chi_{t \geq 0}(t) \sqrt{\frac{2}{\pi \sigma_{r}^{2}}} e^{-t^{2} / 2 \sigma_{r}^{2}}$ which satisfies condition (17) and where $\sigma_{r}=0.5 \mu \mathrm{s}$

- Define $\left\{V_{i}\right\}_{n+1}=\left\{W_{i}\right\}_{n+1} /\left\|\left\{W_{i}\right\}_{n+1}\right\|_{L^{\infty}\left(\left[0, T_{f}\right]\right)}$

Upon employing a regularized version $\mathbb{J}_{s}$ of the cost function $\mathbb{J}$, which is defined by (23), using convolution with the 


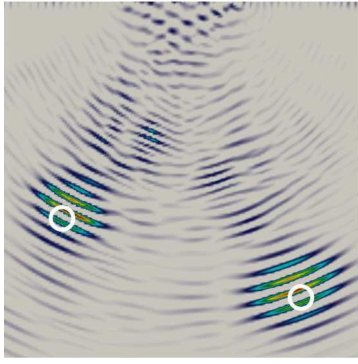

(a) $\hat{\mathbb{T}}_{1}$

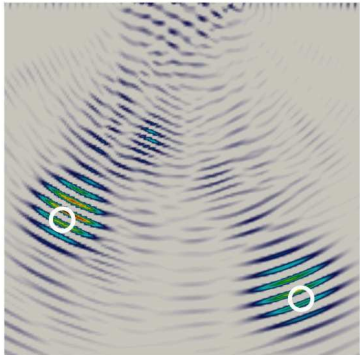

(b) $\hat{\mathbb{T}}_{2}$

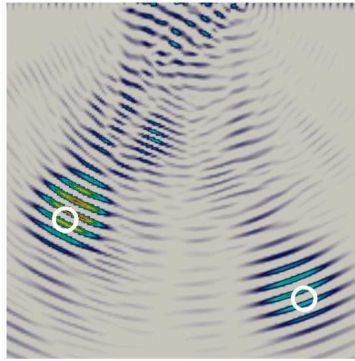

(c) $\hat{\mathbb{T}}_{3}$

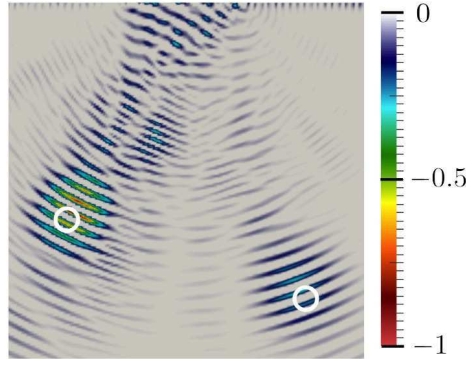

(d) $\hat{\mathbb{T}}_{4}$

Figure 11: Config. 1: Optimally-focusing excitations and iterated indicator functions $\hat{\mathbb{T}}_{n}$.

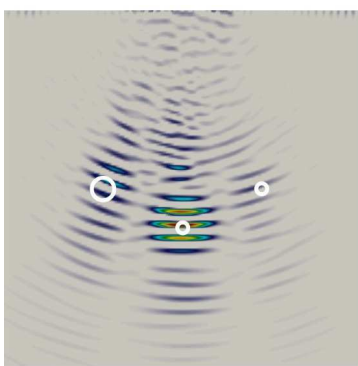

(a) $\hat{\mathbb{T}}_{1}$

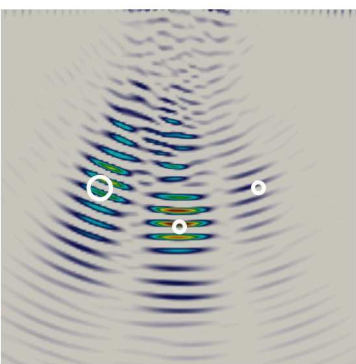

(b) $\hat{\mathbb{T}}_{2}$

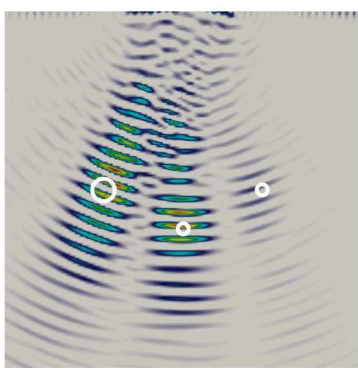

(c) $\hat{\mathbb{T}}_{3}$

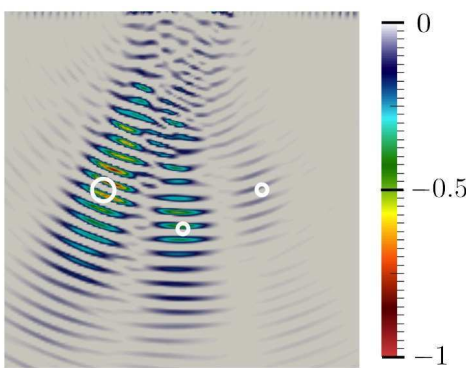

(d) $\hat{\mathbb{T}}_{4}$

Figure 12: Config. 2: Optimally-focusing excitations and iterated indicator functions $\hat{\mathbb{T}}_{n}$.

above positive time domain function $s(t)$, i.e. such that

$$
\mathbb{J}_{s}\left(\varphi^{*} ; T_{f}\right)=\frac{1}{2} \int_{0}^{T_{f}} \sum_{i=1}^{N_{B}} s \star\left|\varphi_{\mid \Gamma_{i}^{c}}^{*}-\varphi_{\mid \Gamma_{i}^{c}}^{\mathrm{obs}}\right|^{2} \mathrm{~d} t,
$$

then according to definition (28-29) of the adjoint state, one notices that $\left(\hat{\boldsymbol{u}}_{n}, \hat{\varphi}_{n}\right) \equiv\left(-\boldsymbol{u}_{n+1},-\varphi_{n+1}\right)$. Therefore, according to (35), one computes for $n \geq 0$ the corresponding topological derivative

$$
\mathbb{T}_{n}\left(\boldsymbol{z} ; \mathcal{D}, T_{f}\right)=R \frac{d}{d t}\left(\boldsymbol{\sigma}\left[\boldsymbol{u}_{n}\right] \underset{\underline{ }}{=} \mathcal{A}(\mathcal{D}): \boldsymbol{\sigma}\left[\hat{\boldsymbol{u}}_{n}\right]+\rho \frac{d \boldsymbol{u}_{n}}{d t} \star \frac{\hat{\boldsymbol{u}}_{n}}{d t}\right)(\boldsymbol{z}),
$$

using polarization tensor (33b), and finally plots its thresholded normalized counterpart $\hat{\mathbb{T}}_{n}$. Figure 10 shows the results obtained for the standard regularized topological derivative $\hat{\mathbb{T}}_{0}$, while iterated indicator functions $\hat{\mathbb{T}}_{n}$ are plotted on Figure 11 and 12.

\subsubsection{Discussion}

Figure 7 and 8 show identification results using piezoelectric transducer with varying number $N_{B}$ of piezoelectric bars. The cases $N_{B}=1$, i.e. Fig. 7 a and Fig. 8a, constitute typical examples illustrating the problem ill-posedness, which is described in Section 5.2, due to the resulting axial symmetry of the configuration considered. However, as the number of piezoelectric bars increases, the resolution improves and the plotted indicator functions exhibit minima that tend to localize in the area of the actual defects. Moreover, piezoelectric reconstructions converge qualitatively to reference fullfield measurements based results shown on panels $\mathrm{f}$, hence corroborating the heuristic of the extension of the topological 
sensitivity approach to piezoelectric-sensor based measurements. The identification of the out-of-axis cavity of Fig. 8 is deteriorated relatively to Fig. 7, a phenomenon which can be accounted for by the characteristic in-axis transducer emission of elastic energy which is illustrated Fig. 3. Finally, as shown on Figure 9 the reconstruction legibility can be enhanced by introducing an additional cutoff parameter which allows to locate precisely the global minimum of the indicator function.

For the cases of simultaneous identifications of multiple cavity, Figure 10 illustrates the influence of the distance between obstacles and of their relative scattering strengths, a factor which is correlated to the assumed illumination. The two well-separated cavities of Fig. 10a are relatively well located by the global minima of the indicator function, whereas on Fig. 10b the different obstacles are not correctly identified. One may also note the relative spreading of the indicator function of Fig. 10a ; These artefacts may be associated with the adjoint field features and discrepancy from its full-field measurements based counterpart that are described in Section 7.2.

When optimally-focusing excitations are used to probe the media considered, as in Fig. 11 and 12, the identification results are significantly improved. Comparison between indicator functions $\hat{\mathbb{T}}_{0}$ and $\hat{\mathbb{T}}_{1}$ on Fig. 10a and 11a highlights that a single iteration of the proposed method reduces remarkably the previously observed artefacts. Concerning iterated illuminations with corresponding indicator functions plotted Fig. 11a-d and 12a-d, then global minima tend to localize in the area of the strongest scatterers in accordance with the expected properties of the approach that are discussed in Section 4.3. Note that the geometric spreading of the indicator function increases with iterations, a feature which may be caused by the expected convergence of the iterated adjoint field towards a harmonic solution, a phenomenon which is described in [2] in a slightly different context.

\section{Conclusions}

This study concerns the investigation of an inverse scattering problem revolving around the identification of defects embedded in elastic solids from piezoelectric sensor-based electric measurements. A fundamental piezoelectric reciprocity identity is proven and applied to a specific transducer model. The characteristic features of such inverse problem associated with electric data are discussed and a comparison is drawn with commonly used direct displacement field boundary measurements in full, or partial, waveform. Along the lines of iterative time reversal techniques, a construction procedure of optimally-focusing electric inputs is presented and implemented. In the context of traction-free cavities, extraction of the informations encapsulated in the available measurements is investigated by way of an adjoint-field based topological sensitivity approach, which uses the derived reciprocity relations, leading to the construction and the non-iterative computation of a defect indicator function. The proposed approach is deployed within the framework of a spectral finite-elements computational platform in order to assess its capabilities and performances on 2D examples. For

completeness, the comparison is made with topological sensitivity-based reconstructions featuring full-field boundary measurements.

Theoretical work remains to be done to provide proper mathematical backing to the interpretation of the indicator 
function (32b), as well as to characterize the eigensystem of the iterated time reversal operator introduced in Theorem 4.1. Future studies will also encompass practical and computational investigations of the expected straightforward generalization of this work to other types of defects, and extensions of the proposed approach in association with topological sensitivity-based indicator functions developed for anisotropic and heterogeneous elastic solids. Finally, addressing numerically reconstruction problems associated with 3D configurations remains to be done. To do so, the design of a specific algorithm aiming at limiting the memory cost associated with the time-domain convolutions in equation (32b) is needed.

\section{References}

[1] J. D. Achenbach. Reciprocity in elastodynamics. Cambridge Monographs on Mechanics. Cambridge University Press, Cambridge, 2003.

[2] C. Ben Amar and C. Hazard. Time reversal and scattering theory for time-dependent acoustic waves in a homogeneous medium. IMA J. Appl. Math., 76:938-955, 2011.

[3] H. Ammari and H. Kang. Polarization and moment tensors with applications to inverse problems and effective medium theory, volume 162 of Applied Mathematical Sciences. Springer, Berlin, 2007.

[4] T. Arens. Linear sampling methods for 2D inverse elastic wave scattering. Inverse Problems, 17:1445-1464, 2001.

[5] B. A. Auld. General electromechanical reciprocity relations applied to the calculation of elastic wave scattering coefficients. Wave Motion, 1:3-10, 1979.

[6] C. Bellis and M. Bonnet. A FEM-based topological sensitivity approach for fast qualitative identification of buried cavities from elastodynamic overdetermined boundary data. International Journal of Solids and Structures, 47(9):1221-1242, 2010.

[7] C. Bellis and M. Bonnet. Qualitative identification of cracks using 3D transient elastodynamic topological derivative: Formulation and FE implementation. Comput. Methods Appl. Mech. Engrg., 253:89-105, 2013.

[8] C. Bellis and S. Imperiale. Dynamical one-dimensional models of passive piezoelectric sensors. Math. Mech. Solids, pages 1-26, 2012.

[9] A. Bendali and Y. Boubendir. Non-overlapping domain decomposition method for a nodal finite element method. Numerische Mathematik, 103(4):515-537, 2006.

[10] M. Bonnet. Topological sensitivity for 3D elastodynamics and acoustic inverse scattering in the time domain. Comput. Methods Appl. Mech. Engrg., 195:5239-5254, 2006.

[11] M. Bonnet and A. Constantinescu. Inverse problems in elasticity. Inverse Problems, 21:R1-R50, 2005. 
[12] M. Bonnet and B. B. Guzina. Sounding of finite solid bodies by way of topological derivative. Int. J. Num. Meth. in Eng., 61:2344-2373, 2004.

[13] H. D. Bui, A. Constantinescu, and H. Maigre. Numerical identification of linear cracks in 2 D elastodynamics using the instantaneous reciprocity gap. Inverse Problems, 20:993-1001, 2004.

[14] F. Cakoni and D. Colton. Qualitative methods in inverse scattering theory. Springer, Berlin, 2006.

[15] A. Charalambopoulos, A. Kirsch, K. A. Anagnostopoulos, D. Gintides, and K. Kiriaki. The factorization method in inverse elastic scattering from penetrable bodies. Inverse Problems, 23:27-51, 2007.

[16] I. Chikichev and B. B. Guzina. Generalized topological derivative for the navier equation and inverse scattering in the time domain. Comp. Meth. Appl. Mech. Engng., 197:4467-4484, 2008.

[17] G. Cohen. Higher-order numerical methods for transient wave equations. Springer, 2001.

[18] E. Demaldent and S. Imperiale. Perfectly matched transmission problems with absorbing layers: Application to anisotropic acoustics. Submitted, 2012.

[19] N. Dominguez, V. Gibiat, and Y. Esquerre. Time domain topological gradient and time reversal analogy: an inverse method for ultrasonic target detection. Wave Motion, 42(1):31-52, 2005.

[20] L. L. Foldy and H. Primakoff. A general theory of passive linear electroacoustic transducers and the electroacoustic reciprocity theorem. I. J. Acoust. Soc. Am., 17:109-120, 1945.

[21] S. Garreau, P. Guillaume, and M. Masmoudi. The topological asymptotic for pde systems: the elasticity case. SIAM J. Control Optim., 39:1756-1778, 2001.

[22] B.B. Guzina and M. Bonnet. Topological derivative for the inverse scattering of elastic waves. Quart. J. Mech. Appl. Math., 57:161-179, 2004.

[23] E. J. Haug, K. K. Choi, and V. Komkov. Design Sensitivity Analysis of Structural Systems. Academic Press, 1986.

[24] C. Hazard and K. Ramdani. Slective acoustic focusing using time-harmonic reversal mirrors. SIAM J. Appl. Math., 64:1657-1676, 2004.

[25] T. Ikeda. Fundamentals of piezoelectricity. Oxford University Press, 1990.

[26] S. Imperiale. Etude mathématique et numérique de capteurs piézoélectriques. PhD thesis, Université Paris Dauphine, France, 2012.

[27] S. Imperiale and P. Joly. Mathematical and numerical modelling of piezoelectric sensors. ESAIM: Mathematical Modelling and Numerical Analysis, 46:875-909, 2012. 
[28] D. Komatitsch and J. Tromp. Introduction to the spectral element method for three-dimensional seismic wave propagation. Geophysical Journal International, 139(3):806-822, 2002.

[29] R. Lerch. Simulation of piezoelectric devices by two-and three-dimensional finite elements. IEEE Trans. Ultra. Ferr. Freq. Cont., 37:233-247, 2002.

[30] J. Y. Li. Uniqueness and reciprocity theorems for linear thermo-electro-magneto-elasticity. Quart. J. Mech. Appl. Math., 56:35-43, 2003.

[31] S. Nintcheu Fata and B. B. Guzina. A linear sampling method for near-field inverse problems in elastodynamics. Inverse Problems, 20:713-736, 2004.

[32] W. Nowacki. A reciprocity theorem for coupled mechanical and thermoelectric fields in piezoelectric crystals. In Proceedings of Vibration Problems, volume 6, pages 3-11, Warsaw, 1965.

[33] A. A. Oberai, G. R. Feijoo, and P.E. Barbone. Lanczos-iterated time reversal. J. Acoust. Soc. Am. Exp. Let., 125:70-76, 2009.

[34] R. Pike and P. Sabatier. Scattering: Scattering and Inverse Scattering in Pure and Applied Science. Academic Press, 2002.

[35] R. E. Plessix, Y. H. De Roeck, and G. Chavent. Waveform inversion of reflection seismic data for kinematic parameters by local optimization. SIAM J. Sci. Comput., 20:1033-1052, 1999.

[36] C. Prada and M. Fink. Eigenmodes of the time reversal operator: A solution to selective focusing in multiple target media. Wave Motion, 20:151-163, 1994.

[37] C. Prada, S. Manneville, D. Spoliansky, and M. Fink. Decomposition of the time reversal operator: Application to detection and selective focusing on two scatterers. J. Acoust. Soc. Am., 99:2067-2076, 1996.

[38] C. Prada, J. L. Thomas, and M. Fink. The iterative time reversal process: Analysis of the convergence. J. Acoust. Soc. Am., 97:62-71, 1995.

[39] I. T. Rekanos, T. V. Yioultsis, and T. D. Tsiboukis. Inverse scattering using the finite element method and a nonlinear optimization technique. IEEE Transactions on microwave theory and techniques, 47:336-344, 1999.

[40] D. Royer and E. Dieulesaint. Elastic waves in solids I. Free and guided propagation. Springer, 2000.

[41] D. Royer and E. Dieulesaint. Elastic waves in solids II. Generation, acousto-optic interaction, applications. Springer, 2000.

[42] Andrieux S., A. Ben Abda, and Bui H. D. Reciprocity principle and crack identification. Inverse Problems, 15:59-65, 1999. 
[43] J. Salençon. Handbook of continuum mechanics: General concepts, thermoelasticity. Springer, 2001.

[44] L. Schmerr Jr and S. J. Song. Ultrasonic nondestructive evaluation systems. Springer, 2007.

[45] J. Sokolowski and A. Zochowski. On the topological derivative in shape optimization. SIAM J. Control Optim., 37:1251-1272, 1999. 